\title{
RISK-SENSITIVE MARKOV CONTROL PROCESSES
}

\author{
YUN SHEN*, WILHELM STANNAT ${ }^{\dagger}$, AND KLAUS OBERMAYER ${ }^{\ddagger}$
}

\begin{abstract}
We introduce a general framework for measuring risk in the context of Markov control processes with risk maps on general Borel spaces that generalize known concepts of risk measures in mathematical finance, operations research and behavioral economics. Within the framework, applying weighted norm spaces to incorporate also unbounded costs, we study two types of infinite-horizon risk-sensitive criteria, discounted total risk and average risk, and solve the associated optimization problems by dynamic programming. For the discounted case, we propose a new discount scheme, which is different from the conventional form but consistent with the existing literature, while for the average risk criterion, we state Lyapunov-like stability conditions that generalize known conditions for Markov chains to ensure the existence of solutions to the optimality equation.
\end{abstract}

Key words. Markov control processes, Poisson equation, Bellman equation, risk-sensitive control, risk measures, stability of nonlinear operators, Doeblin's condition, Lyapunov Stability

AMS subject classifications. 60J05, 93E20, 93C55, 47H07, 91B06

1. Introduction. Markov control processes (MCPs, see e.g. [28, 29] and [36] under the name Markov decision processes) are widely applied to model sequential decision making problems of agents. The induced optimal control problem is to find the best policy that minimizes the expected total costs. The core of the MCP-framework consists of two objective descriptions of some mechanism of the environment: transition probabilities of switching states when performing actions, and immediate outcomes (rewards or costs) obtained at states by executing actions. Facing the same environment, however, different agents might have different policies. Therefore, in many applications, it is important to also incorporate the subjective perceptions of an agent into the MCP-framework. The subjective outcomes are usually modeled by utility functions (see e.g. 24]), which can be easily incorporated by simply replacing the immediate outcome with its utility, whereas the subjective transition probabilities require a more sophisticated mathematical framework. They are commonly incorporated in the risk, which is caused by an uncertain environment.

Coherent/convex risk measures (CRMs) 2, 21 have been widely employed to model subjective probabilities in mathematical finance since the last decade. Several works (see e.g. 37, 20, 8, 39, 9] and references therein) extend CRMs to temporal structures in various setups, where they consider mainly finite-horizon problems. On the contrary, in the literature of MCPs, while the infinite-horizon risksensitive optimal control problems are studied, they apply merely the entropic map [1, 3, 4, 16, 12, 19, 26, 33, 5, which is convex and in fact a special type of CRM. All risk measures mentioned in the above literature are coherent/convex based on the assumption that the agent is supposed to be economically rational and therefore risk-averse. This limits applications in the fields of decision-making under risk and behavioral economics, where more general risk measures (see e.g. [41, 6, 45] and ref-

*Fakultät Elektrotechnik und Informatik, Technische Universität Berlin, Marchstr. 23, 10587, Berlin, Germany (yun@ni.tu-berlin.de).

${ }^{\dagger}$ Institut für Mathematik, and Bernstein Center for Computational Neuroscience, Technische Universität Berlin, Straße des 17. Juni 136, 10623, Berlin, Germany (stannat@math.tu-berlin.de). The work of this author was supported by the BMBF, FKZ01GQ1001B.

${ }^{\ddagger}$ Fakultät Elektrotechnik und Informatik, and Bernstein Center for Computational Neuroscience, Technische Universität Berlin, Marchstr. 23, 10587, Berlin, Germany (oby@ni.tu-berlin.de). The work of this author was supported by the BMBF (Bersteinfokus Lernen TP1), 01GQ0911. 
erences therein) are applied, since human beings are not always risk-averse. However, the models in these fields can only be applied to one-step decision making problems.

To overcome the limitations mentioned above, (i) we extend the definition of CRMs to include the risk measures considered also in behavioral economics; (ii) we apply a constructive approach (see (1.4) below) which maintains the Markov property that is necessary for the existence of stationary optimal policies for two infinite-horizon objectives, albeit less general than the risk maps used in 39, 38.

More specifically, three types of objectives are usually considered in the literature of MCPs: finite-stage, discounted and average cost, depicted as

$$
S_{T}:=\sum_{t=0}^{T} c\left(X_{t}, A_{t}\right), \quad S_{\alpha}:=\sum_{t=0}^{\infty} \alpha^{t} c\left(X_{t}, A_{t}\right), \quad \text { and } \quad S_{A}:=\limsup _{T \rightarrow \infty} \frac{1}{T} S_{T},
$$

where $X_{t}$ and $A_{t}$ are state and action at time $t$ respectively, $c$ denotes the cost function and $\alpha \in[0,1)$ is the discount factor. Given the initial state $X_{0}=x$, the optimization problem is then to minimize the expected objective

$$
\inf _{\boldsymbol{\pi}} \mathbb{E}^{\boldsymbol{\pi}}\left[\mathcal{S} \mid X_{0}=x\right]
$$

by selecting a Markov policy $\boldsymbol{\pi}=\left[\pi_{0}, \pi_{1}, \ldots\right]$, where $\mathcal{S}$ is $S_{T}, S_{\alpha}$ or $S_{A}$. We are mainly interested in the risk-sensitive extensions of two infinite-horizon objectives, i.e., the discounted and average one. We notice that the discounted objective (while other two objectives can be dealt with analogously) can be decomposed as follows,

$$
\mathbb{E}_{X_{0}}^{\pi}\left[S_{\alpha}\right]=c^{\pi_{0}}\left(X_{0}\right)+\alpha \mathbb{E}_{X_{0}}^{\pi_{0}}\left[c^{\pi_{1}}\left(X_{1}\right)+\alpha \mathbb{E}_{X_{1}}^{\pi_{1}}\left[c^{\pi_{2}}\left(X_{2}\right)+\ldots\right]\right]
$$

where $\mathbb{E}_{X}^{\pi}[\cdot]$ denotes conditional expectation at state $X$ under policy $\pi$. We replace the risk-neutral conditional expectation with risk maps $\mathcal{R}_{X}^{\pi}[\cdot]$ similar to the Markov risk measures defined in [39, 38, and obtain the risk-sensitive objective

$$
J_{\alpha}^{\pi}=c^{\pi_{0}}\left(X_{0}\right)+\alpha \mathcal{R}_{X_{0}}^{\pi_{0}}\left[c^{\pi_{1}}\left(X_{1}\right)+\alpha \mathcal{R}_{X_{1}}^{\pi_{1}}\left[c^{\pi_{2}}\left(X_{2}\right)+\ldots\right]\right] .
$$

With the generalized risk measures and constructed risk maps, we provide a unified treatment in the context of MCPs to infinite-horizon risk-sensitive optimal control problems considered in various fields, e.g. optimal control, operations research, finance and behavioral economics. Using weighted norm spaces, we can for the first time incorporate unbounded costs in risk-sensitive MCPs also. We prove that two types of objectives, the discounted total risk and the average risk, can be optimized with $d y$ namic programming algorithms under proper assumptions. For the case of discounted risk, we apply a new discount scheme which is different from the conventional form but consistent with the one applied in [38] where coherent risk measures are considered. For the average case, we state sufficient conditions, which generalize Lyapunov-type conditions from the literature of Markov chains (see e.g. 34]), to ensure the existence of solutions to the associated optimality equation.

The paper is organized as follows. In Section 2 we introduce our concept of risk measures in the context of MCPs on Borel spaces, generalizing CRMs considered in the mathematical finance, in order to also include the family of risk measures considered in behavioral economics. In Section 3 we extend our definition of risk measures to a Markovian temporal structure and call them accordingly risk maps, whose properties are also investigated. In Section 4, we consider risk maps in the MCP-framework by adding control parameters. We demonstrate in Subsection 4.2 how to explicitly 
construct complex risk maps by combining simpler ones, e.g., risk-averse and riskseeking maps, followed by a discussion of examples covered by our framework in Subsection 4.3. The induced infinite-horizon risk-sensitive objectives, including the discounted and average risk, are optimized under assumptions in Subsection 5.1 and 5.2. Finally, in Section 6. we present one example with one risk map and prove that the proposed sufficient conditions for average risk are satisfied.

2. Preliminaries. In this section, we introduce the framework of Markov control processes (MCPs) and risk measures, where in the first part, we mostly follow the notations of Hernández-Lerma \& Lasserre (1999) [29]. We clarify some concepts before stating setups. A Borel space is a Borel subset of a complete separable metric space. If $\mathbf{X}$ is a Borel space, its Borel $\sigma$-algebra is denoted by $\mathcal{B}(\mathbf{X})$. Let $\mathbf{X}$ and $\mathbf{Y}$ be two Borel spaces. A stochastic kernel on $\mathbf{X}$ given $\mathbf{Y}$ is a function $\psi(B \mid y), B \in \mathcal{B}(\mathbf{X}), y \in \mathbf{Y}$ such that i) $\psi(\cdot \mid y)$ is a probability measure on $\mathcal{B}(\mathbf{X})$ for every fixed $y \in \mathbf{Y}$, and ii) $\psi(B \mid \cdot)$ is a measurable function on $\mathbf{Y}$ for every fixed $B \in \mathcal{B}(\mathbf{X})$.

2.1. Markov Control Processes. A Markov control process, $(\mathbf{X}, \mathbf{A},\{\mathbf{A}(x) \mid x \in$ $\mathbf{X}\}, Q, c)$, consists of the following components: state space $\mathbf{X}$ and action space $\mathbf{A}$, which are Borel spaces; the feasible action set $\mathbf{A}(x)$, which is a nonempty Borel space of $\mathbf{A}$, for a given state $x \in \mathbf{X}$; the transition model $Q(B \mid x, a), B \in \mathcal{B}(\mathbf{X}),(x, a) \in \mathbf{K}$ : a stochastic kernel on $\mathbf{X}$ given $\mathbf{K}$, where $\mathbf{K}$ denotes the set of feasible state-action pairs $\mathbf{K}:=\{(x, a) \mid x \in \mathbf{X}, a \in \mathbf{A}(x)\}$, which is a Borel subset of $\mathbf{X} \times \mathbf{A}$; and the cost function $c: \mathbf{K} \rightarrow \mathbb{R}, \mathcal{B}(\mathbf{K})$-measurable. Random variables are denoted by capital letters, e.g. $X_{t}$ and $A_{t}$, whereas realizations of the random variables are denoted by normal letters, e.g. $x_{t}$ and $a_{t}$.

We consider in this paper Markov policies, $\boldsymbol{\pi}=\left[\pi_{0}, \pi_{1}, \pi_{2}, \ldots\right]$, where each singlestep policy $\pi_{t}\left(\cdot \mid x_{t}\right)$, which denotes the probability of choosing action $a_{t}$ at $x_{t},\left(x_{t}, a_{t}\right) \in$ $\mathbf{K}$, is Markov (independent of the states and actions before $t$ ) and, therefore, a stochastic kernel on $\mathbf{A}$ given $\mathbf{X}$. We use the bold typeface to represent a sequence of policies while using normal typeface for a single-step policy. Let $\Delta$ denote the set of all stochastic kernels on $\mathbf{A}$ given $\mathbf{X}, \mu$, such that $\mu(\mathbf{A}(x) \mid x)=1$ and $\Pi_{M}$ denotes the set of all Markov policies. Thus $\Pi_{M}=\Delta^{\infty}$. A policy $f \in \Delta$ is deterministic if for each $x \in \mathbf{X}$, there exists some $a \in \mathbf{A}(x)$ such that $f(\{a\} \mid x)=1$. Let $\Delta_{D} \subset \Delta$ denote the set of all deterministic single-step policies. A policy $\boldsymbol{\pi}$ is said to be stationary, if $\boldsymbol{\pi}=\pi^{\infty}$ for some $\pi \in \Delta$. For each $x \in \mathbf{X}$ and single-step policy $\pi \in \Delta$, define

$$
c^{\pi}(x):=\int_{\mathbf{A}(x)} c(x, a) \pi(\mathrm{d} a \mid x), P^{\pi}(B \mid x):=\int_{\mathbf{A}(x)} Q(B \mid x, a) \pi(\mathrm{d} a \mid x), B \in \mathcal{B}(\mathbf{X}) .
$$

There are usually three types of objectives used in the literature of MCPs: finitestage, discounted and average cost, depicted as

$$
S_{T}:=\sum_{t=0}^{T} c\left(X_{t}, A_{t}\right), \quad S_{\alpha}:=\sum_{t=0}^{\infty} \alpha^{t} c\left(X_{t}, A_{t}\right), \quad \text { and } \quad S_{A}:=\limsup _{T \rightarrow \infty} \frac{1}{T} S_{T}
$$

where $\alpha \in[0,1)$ denotes the discount factor. Suppose we start from one given state $X_{0}=x$. The optimization problem is then to minimize the expected objective

$$
\inf _{\boldsymbol{\pi} \in \Pi_{M}} \mathbb{E}^{\boldsymbol{\pi}}\left[\mathcal{S} \mid X_{0}=x\right]
$$

by selecting a policy $\pi$, where $\mathcal{S}$ is $S_{T}, S_{\alpha}$ or $S_{A}$. We notice that the finite-stage objective function (while other two objectives can be dealt with analogously) can be 
decomposed as follows,

$$
\mathbb{E}_{X_{0}}^{\boldsymbol{\pi}}\left[S_{T}\right]=c^{\pi_{0}}\left(X_{0}\right)+\mathbb{E}_{X_{0}}^{\pi_{0}}\left[c^{\pi_{1}}\left(X_{1}\right)+\mathbb{E}_{X_{1}}^{\pi_{1}}\left[c^{\pi_{2}}\left(X_{2}\right)+\ldots+\mathbb{E}_{X_{T-1}}^{\pi_{T-1}}\left[c^{\pi_{T}}\left(X_{T}\right)\right] \ldots\right]\right]
$$

where $\mathbb{E}_{X_{t}}^{\pi_{t}}\left[v\left(X_{t+1}\right)\right]:=\int v\left(X_{t+1}\right) P^{\pi_{t}}\left(d X_{t+1} \mid X_{t}\right)$ denotes the conditional expectation of the function $v$ of the successive state $X_{t+1}$ given current state $X_{t}$. Obviously, the conditional expectation plays the key role in the calculation of all three objectives.

In order to incorporate risk, we directly replace the expectation $\mathbb{E}_{X_{t}}^{\pi_{t}}$ with a risk map $\mathcal{R}_{X_{t}}^{\pi_{t}}$ that is similar to the risk mapping defined in [39] and will be formally introduced in Section 4. With the replacement, we obtain the risk-sensitive objective

$$
J_{T}^{\boldsymbol{\pi}}=c^{\pi_{0}}\left(X_{0}\right)+\mathcal{R}_{X_{0}}^{\pi_{0}}\left[c^{\pi_{1}}\left(X_{1}\right)+\mathcal{R}_{X_{1}}^{\pi_{1}}\left[c^{\pi_{2}}\left(X_{2}\right)+\ldots+\mathcal{R}_{X_{T-1}}^{\pi_{T-1}}\left[c^{\pi_{T}}\left(X_{T}\right)\right] \ldots\right]\right] .
$$

The other two objectives will be defined analogously and discussed in Section 5

Remark. Comparing with 38] where dynamic risk measures depending on the whole history are allowed, we apply here a less general and Markovian type of risk measures which depends only on the current state. It is due to the motivation that (i) the underlying structure is Markovian, and (ii) for optimizing infinite-horizon objectives, it becomes computationally infeasible if risk measures are dependent on the whole history.

2.2. Risk Measures. In order to include risk measures considered also in behavioral economics, we introduce a generalized version of risk measures which are originally defined in [2, 21]. Consider a probability space $(\mathbf{X}, \mathcal{B}(\mathbf{X}))$ under some probability measure $\varphi$. Let $\mathscr{L}$ be a vector space of $\mathcal{B}(\mathbf{X})$-measurable real-valued functions and $\mathscr{B}$ be the space of all bounded $\mathcal{B}(\mathbf{X})$-measurable real-valued functions. We assume $\mathscr{L} \supset \mathscr{B}$. The partial ordering " $\leq$ " in $\mathscr{L}$ is defined as: $v \leq u$, if $v(x) \leq u(x)$ for all $x \in \mathbf{X}$. For convenience, we write $u \in \mathbb{R}$ if $u$ is a constant real-valued function. Thus $u \in \mathscr{L}$, which will be specified in Section 3.2

Definition 2.1. A mapping $\nu: \mathscr{L} \rightarrow \mathbb{R} \cup\{\infty\}$ is said to be a risk measure if

(I) (Monotonicity) $\nu(v) \leq \nu(u)$, whenever $v \leq u \in \mathscr{L}$;

(II) (Translation invariance) $\nu(v+u)=\nu(v)+u$, for any $u \in \mathbb{R}$;

(III) (Centralization) $\nu(0)=0$.

Moreover, $\nu$ is called real-valued if $\nu(v) \in \mathbb{R}$ for all $v \in \mathscr{L}$.

Remark. Within the economic context, $v$ and $u$ are usually considered as random variables, which are used to model the uncertain costs in the future. Monotonicity reflects the intuition that if the cost of one case is higher than the cost of another case, the risk of the case must be higher than that of the other one. Under the axiom of translation invariance, the sure cost $u$ (equal everywhere in the state space $\mathbf{X}$ ) in the future, which can be viewed as a constant function, is considered as a sure cost at current time point. The axiom of centralization sets the reference point to be 0 , i.e., there is no risk if there is no cost.

Definition 2.2. A risk measure $\nu$ is called

- convex, if for all $\alpha \in[0,1], v, u \in \mathscr{L}, \nu(\alpha v+(1-\alpha) u) \leq \alpha \nu(v)+(1-\alpha) \nu(u)$;

- concave, if for all $\alpha \in[0,1], v, u \in \mathscr{L}, \nu(\alpha v+(1-\alpha) u) \geq \alpha \nu(v)+(1-\alpha) \nu(u)$;

- homogeneous, if for all $\lambda \in \mathbb{R}_{+}$and $v \in \mathscr{L}, \nu(\lambda v)=\lambda \nu(v)$;

- coherent, if $\nu$ is convex and homogeneous.

Remark. Comparing with the risk measures defined in finance [2, 21, neither convexity nor coherency is required. We will see in Section 4.2 that convex risk measures correspond to the case that the agent is risk-averse. However, in some 
problems, especially in modeling real human behaviors, mixed risk-preference (riskaverse at some states while risk-seeking at other states) is also a possible strategy. For instance, at gambling, some people are risk-averse when losing money but risk-seeking when winning money. Therefore, we require neither coherence nor convexity.

3. Risk Maps without Control. Before applying risk maps in the MCPframework, we first define risk maps without control on Markov chains and investigate their properties. We refer to 39 for conditional risk maps under more general settings. Consider a time-homogenous Markov chain with state space $\mathbf{X}$ and transition kernel $P$.

Definition 3.1. A mapping $\mathcal{R}(x, v): \mathbf{X} \times \mathscr{L} \rightarrow \mathbb{R} \cup\{\infty\}$ is said to be a risk map on the Markov chain $P$, if (i) for each $x \in \mathbf{X}, \mathcal{R}_{x}(\cdot):=\mathcal{R}(x, \cdot)$ is a risk measure; and (ii) $\mathcal{R}(\cdot, v) \in \mathscr{L}$ for each $v \in \mathscr{L}$. $\mathcal{R}$ is called real-valued if for each $x \in \mathbf{X}$ and $v \in \mathscr{L}, \mathcal{R}(x, v) \in \mathbb{R}$. $\mathcal{R}$ is called convex (resp. concave, homogenous, coherent) if for all $x \in \mathbf{X}, \mathcal{R}_{x}$ is convex (resp. concave, homogenous, coherent).

Remark. With slight abuse of terminology, $P$ can be viewed as a linear operator such that $P_{x}(v):=\int v(y) P(d y \mid x), v \in \mathscr{L}$. Then the operator $\mathcal{R}$ defined above is a generalization of $P$, maintaining the key properties, monotonicity, translation invariance and centralization. In the following, we view $\mathcal{R}(v)$ as a function on $\mathscr{L}$, for each $v \in \mathscr{L}$. Finally, for two risk maps, $\mathcal{S}, \mathcal{R}$, we write $\mathcal{S} \leq \mathcal{R}$, if $\mathcal{S}(v) \leq \mathcal{R}(v)$ for all $v \in \mathscr{L}$.

3.1. Sub- and Uppermodules. To investigate stability properties of risk maps, we generalize the modules introduced by Delbaen (2000) [13.

Definition 3.2. Let $\mathcal{F}(x, v): \mathbf{X} \times \mathscr{L} \rightarrow \mathbb{R}$ be a map on $\mathbf{X}$ and $\mathscr{L}$ and write $\mathcal{F}_{x}(v):=\mathcal{F}(x, v)$. Then $\mathcal{F}^{\sharp}: \mathbf{X} \times \mathscr{L} \rightarrow \mathbb{R} \cup\{\infty\}$ defined by $\mathcal{F}_{x}^{\sharp}(v):=$ $\sup _{u \in \mathscr{L}}\left\{\mathcal{F}_{x}(v+u)-\mathcal{F}_{x}(u)\right\}, \forall x \in \mathbf{X}$, is called the submodule of $\mathcal{F}$. Let $\mathcal{G}(x, v)$ : $\mathbf{X} \times \mathscr{L} \rightarrow \mathbb{R} \cup\{\infty\}$ be a map on $\mathbf{X}$ and $\mathscr{L}$ and write $\mathcal{G}_{x}(v):=\mathcal{G}(x, v)$. Then

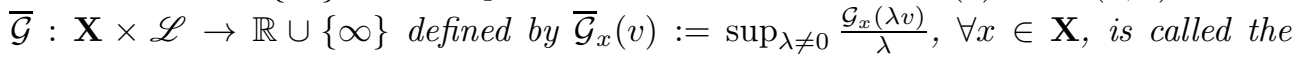
uppermodule of $\mathcal{G}$.

We summarize some properties of sub- and uppermodules of risk maps.

Proposition 3.3. Let $\mathcal{R}$ be a real-valued risk map. Then for each $x \in \mathbf{X}$ (i) $\mathcal{R}_{x}(v) \leq \mathcal{R}_{x}^{\sharp}(v), \forall v \in \mathscr{L}$; (ii) $\mathcal{R}_{x}^{\sharp}$ is a risk measure; (iii) $\mathcal{R}_{x}^{\sharp}$ is sublinear, i.e., $\mathcal{R}_{x}^{\sharp}\left(v+v^{\prime}\right) \leq \mathcal{R}_{x}^{\sharp}(v)+\mathcal{R}_{x}^{\sharp}\left(v^{\prime}\right)$.

Proof. (i) and (ii) are immediate consequences of the construction of the submodule $\mathcal{R}^{\sharp}$ associated with $\mathcal{R}$. It remains to prove (iii). First, for all $v \in \mathscr{L}$ and $x \in \mathbf{X}, \mathcal{R}_{x}^{\sharp}(v)=\sup _{u \in \mathscr{L}}\left\{\mathcal{R}_{x}(v+u)-\mathcal{R}_{x}(u)\right\}=\sup _{u^{\prime} \in \mathscr{L}}\left\{\mathcal{R}_{x}\left(u^{\prime}\right)-\mathcal{R}_{x}\left(u^{\prime}-v\right)\right\}$. Thus, setting $u^{\prime}=v^{\prime}+u$, we have for all $u \in \mathscr{L}$ and $x \in \mathbf{X}$,

$$
\begin{aligned}
& \mathcal{R}_{x}\left(v+v^{\prime}+u\right)-\mathcal{R}_{x}(u) \leq \sup _{u^{\prime} \in \mathscr{L}}\left\{\mathcal{R}_{x}\left(v+u^{\prime}\right)-\mathcal{R}_{x}\left(u^{\prime}\right)+\mathcal{R}_{x}\left(u^{\prime}\right)-\mathcal{R}_{x}\left(u^{\prime}-v^{\prime}\right)\right\} \\
\leq & \sup _{u^{\prime} \in \mathscr{L}}\left\{\mathcal{R}_{x}\left(v+u^{\prime}\right)-\mathcal{R}_{x}\left(u^{\prime}\right)\right\}+\sup _{u^{\prime} \in \mathscr{L}}\left\{\mathcal{R}_{x}\left(u^{\prime}\right)-\mathcal{R}_{x}\left(u^{\prime}-v^{\prime}\right)\right\}=\mathcal{R}_{x}^{\sharp}(v)+\mathcal{R}_{x}^{\sharp}\left(v^{\prime}\right) .
\end{aligned}
$$

Hence $\mathcal{R}^{\sharp}\left(v+v^{\prime}\right) \leq \mathcal{R}^{\sharp}(v)+\mathcal{R}^{\sharp}\left(v^{\prime}\right)$.

Proposition 3.4. Let $\mathcal{R}(x, v)$ be a map on $\mathbf{X} \times \mathscr{L}$ such that for each $x \in \mathbf{X}$, $\mathcal{R}(x, \cdot)$ is a risk measure. Then for all $x \in \mathbf{X}$, (i) $\mathcal{R}_{x}(v) \leq \overline{\mathcal{R}}_{x}(v), \forall v \in \mathscr{L}$; (ii) $\overline{\mathcal{R}}_{x}$ is a risk measure; (iii) $\overline{\mathcal{R}}_{x}$ is homogeneous.

Proof. Similar to their counterparts for submodule, (i) and (ii) are immediate consequences of the construction of the uppermodule $\overline{\mathcal{R}}$ associated with $\mathcal{R}$. For the 
proof of (iii), note that for any $\beta>0$,

$$
\overline{\mathcal{R}}_{x}(\beta v)=\sup _{\lambda \neq 0} \frac{\mathcal{R}_{x}(\lambda \beta v)}{\lambda}=\sup _{\lambda \beta \neq 0} \beta \frac{\mathcal{R}_{x}(\lambda \beta v)}{\beta \lambda}=\beta \sup _{\lambda \neq 0} \frac{\mathcal{R}_{x}(\lambda v)}{\lambda}=\beta \overline{\mathcal{R}}_{x}(v) .
$$

Proposition 3.5. Let $\mathcal{R}$ be a real-valued risk map. Then $\forall x \in \mathbf{X}, \overline{\mathcal{R}}_{x}(v)=$ $\sup _{\lambda>0} \frac{\mathcal{R}_{x}^{\sharp}(\lambda v)}{\lambda}$ and $\overline{\mathcal{R}}_{x}$ is coherent. Furthermore, if $\mathcal{R}$ is coherent, then $\overline{\mathcal{R}^{\sharp}}=\mathcal{R}$.

Proof. (i) First we show that $-\lambda^{-1} \mathcal{R}_{x}^{\sharp}(-\lambda v) \leq \lambda^{-1} \mathcal{R}_{x}^{\sharp}(\lambda v)$ for each $\lambda \in \mathbb{R}_{+}$ and $v \in \mathscr{L}$. Indeed, without loss of generality, assume that $R_{x}^{\sharp}(-\lambda v)<\infty$. Then by sublinearity, $0=\mathcal{R}_{x}^{\sharp}(0)=\mathcal{R}_{x}^{\sharp}(\lambda v-\lambda v) \leq \mathcal{R}_{x}^{\sharp}(\lambda v)+\mathcal{R}_{x}^{\sharp}(-\lambda v)$ implies that $-\lambda^{-1} \mathcal{R}_{x}^{\sharp}(-\lambda v) \leq \lambda^{-1} \mathcal{R}_{x}^{\sharp}(\lambda v)$. Thus $\overline{\mathcal{R}}_{x}^{\sharp}(v)=\sup _{\lambda>0} \frac{\mathcal{R}_{x}^{\sharp}(\lambda v)}{\lambda}, \forall x \in \mathbf{X}$. $\overline{\mathcal{R}}_{x}^{\sharp}$ is coherent, since $\overline{\mathcal{R}}_{x}$ is both sublinear and homogeneous.

(ii) Suppose $\mathcal{R}$ is coherent. Then $\mathcal{R}(v+u)-\mathcal{R}(u) \leq \mathcal{R}(v)$ for all $v, u \in \mathscr{L}$. Hence, $\mathcal{R}^{\sharp} \leq \mathcal{R}$. Together with Proposition $3.3(\mathrm{i}) \mathcal{R} \leq \mathcal{R}^{\sharp}$, we have $\mathcal{R}^{\sharp}=\mathcal{R}$. Since $\mathcal{R}$ is homogenous, $\overline{\mathcal{R}^{\sharp}}=\mathcal{R}$.

Proposition 3.6. Let $\mathcal{R}$ be a real-valued risk map. Then $\left|\mathcal{R}_{x}(v+u)-\mathcal{R}_{x}(u)\right| \leq$ $\overline{\mathcal{R}}_{x}^{\sharp}(|v|)$ for all $v, u \in \mathscr{L}$ and $x \in \mathbf{X}$.

Proof. By Proposition 3.3 and 3.4, for each $x \in \mathbf{X}, \mathcal{R}_{x}(v+u)-\mathcal{R}_{x}(u) \leq \overline{\mathcal{R}}_{x}(v) \leq$ $\overline{\mathcal{R}}_{x}^{\sharp}(|v|)$. On the other hand, $\mathcal{R}_{x}(u)-\mathcal{R}_{x}(u+v) \leq \overline{\mathcal{R}}_{x}(-v) \leq \overline{\mathcal{R}}_{x}(|v|)$.

Remark. For any real-valued risk map $\mathcal{R}$, its sub-upper-module $\overline{\mathcal{R}^{\sharp}}$ satisfying the three axioms of risk measures is in fact its coherent upper bound. In following sections, we shall apply this upper bound to control the growth speed of iterations of $\mathcal{R}$ (see Assumption 3.1).

3.2. Weighted Norm. We now specify the functional space $\mathscr{L}$ used in this paper. Suppose $w: \mathbf{X} \rightarrow[1, \infty)$ is a given measurable function. Consider the $w$-norm

$$
\|u\|_{w}:=\sup _{x \in \mathbf{X}} \frac{|u(x)|}{w(x)} .
$$

Let $\mathscr{B}_{w}$ be the space of real-valued $w$-bounded $\mathcal{B}(\mathbf{X})$-measurable functions. It is obvious that the bounded functional space $\mathscr{B} \subset \mathscr{B}_{w}$. Let $\mu$ be a signed measure on $\mathcal{B}(\mathbf{X})$. Define $\|\mu\|_{w}:=\sup _{\|u\|_{w} \leq 1}\left|\int_{\mathbf{X}} u d \mu\right|=\int_{\mathbf{X}} w \mathrm{~d}|\mu| \geq\|\mu\|_{T V}$. Denote by $\mathscr{M}\left(\right.$ resp. $\mathscr{M}_{w}$ ) the space of all (resp. $w$-)bounded signed measures on $\mathcal{B}(\mathbf{X})$. Thus $\mathscr{M}_{w} \subset \mathscr{M}$. More discussions about the $w$-norm space with applications in MCPs are referred to $[29$.

Proposition 3.7. Let $\mathcal{R}$ be a real-valued risk map. Suppose there exists a $\bar{w} \in \mathbb{R}_{+}$such that $\overline{\mathcal{R}}_{x}^{\sharp}(w) \leq \bar{w} \cdot w(x), \forall x \in \mathbf{X}$. Then $\|\mathcal{R}(v)-\mathcal{R}(u)\|_{w} \leq \bar{w}\|v-u\|_{w}$, for all $v, u \in \mathscr{B}_{w}$.

Proof. By definition, $|v-u| \leq\|v-u\|_{w} w$. Hence, Proposition 3.6 yields

$$
|\mathcal{R}(v)-\mathcal{R}(u)| \leq \overline{\mathcal{R}^{\sharp}}(|v-u|) \leq \overline{\mathcal{R}^{\sharp}}\left(\|v-u\|_{w} w\right)=\|v-u\|_{w} \overline{\mathcal{R}^{\sharp}}(w)
$$

due to the homogeneity of $\overline{\mathcal{R}^{\sharp}}$. Using the assumption $\overline{\mathcal{R}^{\sharp}}(w) \leq \bar{w} \cdot w$, we obtain that $\|\mathcal{R}(v)-\mathcal{R}(u)\|_{w} \leq \bar{w}\|v-u\|_{w}$.

Corollary 3.8. Under the same assumption of Proposition 3.7. $\mathcal{R}(v) \in \mathscr{B}_{w}$, for all $v \in \mathscr{B}_{w}$.

We consider the following seminorm that will play a key role in Section 3.3 to investigate the stability of risk maps and the existence of a solution to the Poisson 
equation.

$$
\|v\|_{s, w}:=\sup _{x \neq y} \frac{|v(x)-v(y)|}{d_{w}(x, y)}, \text { where } d_{w}(x, y):=\left\{\begin{array}{ll}
0 & x=y \\
w(x)+w(y) & x \neq y
\end{array} .\right.
$$

This seminorm is originally used by Hairer and Mattingly (2011) 25] to study the ergodicity of Markov chains. In particular, when restricting to the bounded space $\mathscr{B}$, i.e. setting $w \equiv 1$, the seminorm is called span-norm in 27] and Hilbert seminorm in [23. In the following, we restate the Lemma 2.1 in [25] and incorporate its proof for readers' convenience.

Lemma 3.9. $\|v\|_{s, w}=\inf _{c \in \mathbb{R}}\|v+c\|_{w}, \forall v \in \mathscr{B}_{w}$.

Proof. It is obvious that $\|v\|_{s, w} \leq\|v\|_{w}$ and therefore $\|v\|_{s, w} \leq \inf _{c \in \mathbb{R}}\|v+c\|_{w}$. It remains to prove the reverse inequality. Given any $\|v\|_{s, w} \leq 1$, set $c=\inf _{x}\{w(x)-$ $v(x)\}$. Note that for any $x$ and $y, v(x) \leq|v(y)|+|v(x)-v(y)| \leq|v(y)|+w(x)+w(y)$. Hence $w(x)-v(x) \geq-w(y)-|v(y)|$, which implies that $c$ is bounded below and hence $|c|<\infty$. Observe that $v(x)+c \leq v(x)+w(x)-v(x) \leq w(x)$ and

$$
v(x)+c=\inf _{y}\{v(x)+w(y)-v(y)\} \geq \inf _{y}\left\{w(y)-d_{w}(x, y)\|v\|_{s, w}\right\} \geq-w(x) .
$$

Hence $|v(x)+c| \leq w(x)$ as required.

3.3. Poisson Equation and Invariant Risk Measure. In this section, we shall prove (see Theorem 3.14) that under some sufficient conditions (see Assumption 3.1) there exist a solution $(\rho, h) \in \mathbb{R} \times \mathscr{B}_{w}$ to the Poisson Equation for some fixed $c \in \mathscr{B}_{w}$ and a real-valued risk map $\mathcal{R}$ on $\mathscr{B}_{w}$,

$$
c+\mathcal{R}(h)=\rho+h
$$

and invariant risk measure, $\nu$, satisfying

$$
\nu(c+\mathcal{R}(v))=\nu(v)+\rho, \forall v \in \mathscr{B}_{w} .
$$

As in the theory of MCPs, both Poisson equation and invariant risk measure play important roles in studying the stability properties of risk maps and the optimization of the average risk (see Section 5.2).

We apply mainly the same trick used in 25 . Consider an auxiliary weight function $W: \mathbf{X} \rightarrow[0, \infty)$, which is a real-valued $\mathcal{B}(\mathbf{X})$-measurable function and let $w(x)=$ $1+\beta W(x)$ with some positive real number $\beta$.

Assumption 3.1. Let $\mathcal{R}$ be real-valued risk map. There exists a function $W$ : $\mathbf{X} \rightarrow[0, \infty)$ which is $\mathcal{B}(\mathbf{X})$-measurable, constants $K \geq 0, \gamma \in(0,1), \alpha \in(0,1)$, $\alpha_{0} \in(0, \alpha)$ and a real-valued risk measure $\nu$ such that $(i)$

$$
\overline{\mathcal{R}}_{x}(W) \leq \gamma W(x)+K, \forall x \in \mathbf{X}
$$

where $\overline{\mathcal{R}}_{x}^{\sharp}(W)$ is calculated on the space $\mathscr{B}_{w}=\mathscr{B}_{1+\beta W}, \beta:=\alpha_{0} / K$, and (ii)

$$
\inf _{x \in B}\left\{\mathcal{R}_{x}(v)-\alpha \nu(v)-\mathcal{R}_{x}(u)+\alpha \nu(u)\right\} \geq 0
$$

whenever $v \geq u \in \mathscr{B}_{w}$, where $B=\{x \in \mathbf{X}: W(x) \leq R\} \in \mathcal{B}(\mathbf{X})$ for some $R>$ $2 K /(1-\gamma)$.

Remark. If $\mathcal{R}$ is coherent, then $\overline{\mathcal{R}^{\sharp}}=\mathcal{R}$ in (3.3). Specifically, if $\overline{\mathcal{R}^{\sharp}}=P$, given that the transition kernel $P$ is considered also as a linear operator (see the remark 
below Definition 3.1), then $\nu$ in (3.4) is a probability measure and (3.4) is equivalent to $\inf _{x \in B}\left\{P_{x}(A)-\alpha \nu(A)\right\} \geq 0, \forall A \in \mathcal{B}(\mathbf{X})$. Therefore, the above assumption is reduced to the condition used in [25, where it is proven to be in some sense equivalent to the classical geometric ergodicity condition stated in 34 .

In the following, we connect the generalized Doeblin's condition in (3.4) with the classical one by subgradients of risk maps [4]. Define the subgradient at state $x \in \mathbf{X}$ and function $u \in \mathscr{B}_{w}$ for a real-valued risk map $\mathcal{R}$ as follows,

$$
\delta \mathcal{R}_{x}(u):=\left\{\begin{array}{l|l}
g \mid \begin{array}{l}
g \text { is } \mathcal{B}(\mathbf{X}) \text {-measurable and } \int|g| w d P_{x}<\infty \\
\mathcal{R}_{x}(v) \geq \mathcal{R}_{x}(u)+\int g(v-u) d P_{x}, \forall v \geq u \in \mathscr{B}_{w}
\end{array}
\end{array}\right\}
$$

where $P_{x}$ denotes the transition probability measure from state $x \in \mathbf{X}$.

Proposition 3.10. Suppose the transition kernel $P$ satisfies that there exists some positive constant $\beta$, set $B \in \mathcal{B}(\mathbf{X})$ and probability measure $\mu$ such that $\inf _{x \in B}\left\{P_{x}(A)-\beta \mu(A)\right\} \geq 0, \forall A \in \mathcal{B}(\mathbf{X})$. Assume further that there exists $g(x, u) \in$ $\delta \mathcal{R}_{x}(u)$ and positive constant $\epsilon>0$ such that $g(x, u) \geq \epsilon$ for all $x \in B$ and $u \in \mathscr{B}_{w}$. Then (3.4) holds for $\nu=\mu$ and $\alpha=\epsilon \beta$.

Proof. By definition, we have for each $x \in B$ and $u \in \mathscr{B}_{w}$

$$
\mathcal{R}_{x}(v) \geq \mathcal{R}_{x}(u)+\int g(x, u)(v-u) d P_{x} \geq R_{x}(u)+\epsilon \beta \mu(v-u) .
$$

Then setting $\nu=\mu$ and $\alpha=\epsilon \beta$, (3.4) holds.

Remark. Subgradients are originally defined for convex risk maps. For concave risk maps $\mathcal{R}$, however, we can consider its convex counterpart $\tilde{\mathcal{R}}(v):=-\mathcal{R}(-v)$. If $\tilde{\mathcal{R}}$ satisfies the generalized Doeblin's condition with some risk measure $\tilde{\nu}$, then it is easy to see that $\mathcal{R}$ also satisfies the condition with the risk measure $\nu(v):=-\tilde{\nu}(-v)$.

Now we state the contraction theorem under $w$-seminorm.

Theorem 3.11. Suppose Assumption 3.1 holds. Then there exists a $\bar{\alpha} \in[0,1)$ such that $\|\mathcal{R}(v)-\mathcal{R}(u)\|_{s, w} \leq \bar{\alpha}\|v-u\|_{s, w}$, for all $v$ and $u$ in $\mathscr{B}_{w}$.

Proof. Clearly, the assertion is equivalent to $\|\mathcal{R}(v+u)-\mathcal{R}(u)\|_{s, w} \leq \bar{\alpha}\|v\|_{s, w}$, $\forall v, u \in \mathscr{B}_{w}$. Suppose $\|v\|_{s, w} \leq C$. Lemma 3.9 suggests that we can always find a real value $c$ such that $\|v+c\|_{w} \leq C$. Since adding any constant to $v$ will not change the values of both sides of the required inequality, without loss of generality, we assume $\|v\|_{w} \leq C$. Hence, $|v(x)| \leq\|v\|_{w} w(x) \leq C w(x), \forall x \in \mathbf{X}$. By definition and Proposition 3.6 we have $\forall x \in \mathbf{X}$,

$$
\left|\mathcal{R}_{x}(v+u)-\mathcal{R}_{x}(u)\right| \leq \overline{\mathcal{R}}_{x}(|v|) \leq\|v\|_{w} \overline{\mathcal{R}}_{x}(w)=C\left(1+\beta \overline{\mathcal{R}}_{x}(W)\right),
$$

where the equality is obtained by using Proposition 3.4(iii).

We first assume $W(x)+W(y) \geq R$ and set $\gamma_{0}:=\gamma+\frac{2 K}{R}<1$ and $\gamma_{1}:=\frac{2+\beta R \gamma_{0}}{2+\beta R} \in$ $\left(\gamma_{0}, 1\right)$. (3.5) yields,

$$
\begin{aligned}
& \left|\mathcal{R}_{x}(v+u)-\mathcal{R}_{x}(u)-\mathcal{R}_{y}(v+u)+\mathcal{R}_{y}(u)\right| \\
\leq & \left|\mathcal{R}_{x}(v+u)-\mathcal{R}_{x}(u)\right|+\left|\mathcal{R}_{y}(v+u)-\mathcal{R}_{y}(u)\right| \\
\leq & C\left(2+\beta \overline{\mathcal{R}}_{x}(W)+\beta \overline{\mathcal{R}}_{y}(W)\right) \leq C(2+\beta \gamma W(x)+\beta \gamma W(y)+2 \beta K) \\
\leq & C\left(2+\beta \gamma_{0} W(x)+\beta \gamma_{0} W(y)\right) \leq C\left(2 \gamma_{1}+\beta \gamma_{1} W(x)+\beta \gamma_{1} W(y)\right) \\
= & C \gamma_{1} d_{\beta}(x, y),
\end{aligned}
$$

where the last inequality is due to fact that $\frac{2\left(1-\gamma_{1}\right)}{\beta\left(\gamma_{1}-\gamma_{0}\right)}=R \leq W(x)+W(y)$. 
Now consider $W(x)+W(y) \leq R$. Thus $x, y \in B$. Define a new risk map $\tilde{\mathcal{R}}_{x}(v):=$ $\frac{1}{1-\alpha} \mathcal{R}_{x}(v)-\frac{\alpha}{1-\alpha} \nu(v)$. It is easy to verify that $\tilde{\mathcal{R}}$ is valid risk map on $B$. In fact, the monotonicity is guaranteed by Assumption [3.1(ii). Hence, by replacing $\mathcal{R}$ with $\tilde{\mathcal{R}}$ (3.5) holds for all $x, y \in B$, which yields

$$
\begin{aligned}
& \left|\mathcal{R}_{x}(v+u)-\mathcal{R}_{x}(u)-\mathcal{R}_{y}(v+u)+\mathcal{R}_{y}(u)\right| \\
= & (1-\alpha)\left|\tilde{\mathcal{R}}_{x}(v+u)-\tilde{\mathcal{R}}_{x}(u)-\tilde{\mathcal{R}}_{y}(v+u)+\tilde{\mathcal{R}}_{y}(u)\right| \\
\leq & (1-\alpha) C\left(2+\beta \overline{\tilde{\mathcal{R}}}_{x}^{\sharp}(W)+\beta \tilde{\tilde{\mathcal{R}}}_{y}^{\sharp}(W)\right) .
\end{aligned}
$$

On the other hand $\overline{\tilde{\mathcal{R}}}_{x}^{\sharp}(W) \leq(1-\alpha)^{-1} \overline{\mathcal{R}}_{x}(W)$, since $W \geq 0$. Hence,

$$
\begin{aligned}
(1-\alpha) C\left(2+\beta \overline{\tilde{\mathcal{R}}}_{x}(W)+\beta \overline{\tilde{\mathcal{R}}}_{y}(W)\right) & \leq 2(1-\alpha) C+\beta C\left(2+\beta \overline{\mathcal{R}}_{x}(W)+\beta \overline{\mathcal{R}}_{y}(W)\right) \\
& \leq 2(1-\alpha) C+\beta C(\gamma W(x)+\gamma W(y)+2 K) .
\end{aligned}
$$

Since $\beta=\alpha_{0} / K$ for some $\alpha_{0} \in(0, \alpha)$, setting $\gamma_{2}:=\left(1-\alpha+\alpha_{0}\right) \vee \gamma \in(0,1)$ yields,

$$
\begin{aligned}
& \left|\mathcal{R}_{x}(v+u)-\mathcal{R}_{x}(u)-\mathcal{R}_{y}(v+u)+\mathcal{R}_{y}(u)\right| \\
\leq & 2 C\left(1-\alpha+\alpha_{0}\right)+C \gamma \beta(W(x)+W(y)) \leq C \gamma_{2} d_{\beta}(x, y) .
\end{aligned}
$$

Hence, setting $\bar{\alpha}:=\gamma_{1} \vee \gamma_{2}<1$, (3.6) and (3.7) imply,

$$
\left|\mathcal{R}_{x}(v+u)-\mathcal{R}_{x}(u)-\mathcal{R}_{y}(v+u)+\mathcal{R}_{y}(u)\right| \leq\|v\|_{s, w} \bar{\alpha} d_{\beta}(x, y) .
$$

LEMma 3.12. Let $\mathcal{T}: \mathscr{B}_{w} \rightarrow \mathscr{B}_{w}$ be an operator satisfying $\mathcal{T}(v+c)=\mathcal{T}(v)+$ $c, \forall v \in \mathscr{B}_{w}, c \in \mathbb{R}$ and define its iteration as $\mathcal{T}^{t}(v):=\mathcal{T}\left(\mathcal{T}^{t}(v)\right), t=2,3, \ldots$ Suppose $\mathcal{T}$ satisfies furthermore that for all $v, u \in \mathscr{B}_{w},(i)\|\mathcal{T}(v)-\mathcal{T}(u)\|_{s, w} \leq \bar{\alpha}\|v-u\|_{s, w}$ for some $\bar{\alpha} \in[0,1)$; and (ii) $\sup _{t>1}\left\|\mathcal{T}^{t}(v)-\mathcal{T}^{t}(u)\right\|_{w} \leq A\|v-u\|_{w}$ with some constant $A \in \mathbb{R}_{+}$. Then for arbitrary probability measure $\mu_{0} \in \mathscr{M}_{w}$ and $v, u \in \mathscr{B}_{w}$,

$$
\lim _{s \rightarrow \infty} \sup _{t \geq s}\left|\mu_{0}\left[\mathcal{T}^{t}(v)-\mathcal{T}^{t}(u)\right]-\mu_{2}\left[\mathcal{T}^{s}(v)-\mathcal{T}^{s}(u)\right]\right|=0
$$

Proof. Let $v_{t}:=\mathcal{T}^{(t)}(v)$ and $u_{t}:=\mathcal{T}^{(t)}(u), t=1,2, \ldots$ For $v, u \in \mathscr{B}_{w}$, without loss of generality, we assume that $\|v-u\| \leq C$, where $C$ is a positive real number. Hence, for any $t \geq s$,

$$
\begin{aligned}
\text { (Lemma 3.9) } & =\sup _{\|v-u\|_{w} \leq C}\left|\mu_{0}\left[\mathcal{T}^{t}(v)-\mathcal{T}^{t}(u)\right]-\mu_{0}\left[\mathcal{T}^{s}(v)-\mathcal{T}^{s}(u)\right]\right| \\
\text { (by (i) }) & \leq \sup _{\left\|v_{1}-u_{1}\right\|_{s, w} \leq \bar{\alpha} C}\left|\mu_{0}\left[\mathcal{T}^{t}(v)-\mathcal{T}^{t}(u)\right]-\mu_{0}\left[\mathcal{T}^{s}(v)-\mathcal{T}^{s}(u)\right]\right| \\
& \leq \sup _{\left\|v_{s}-u_{s}\right\|_{s, w} \leq \bar{\alpha}^{s} C}\left|\mu_{0}\left[\mathcal{T}^{t-s}\left(v_{s}\right)-\mathcal{T}^{t-1}\left(u_{1}\right)\right]-\mu_{0}\left[\mathcal{T}^{s-1}\left(v_{1}\right)-\mathcal{T}^{s-1}\left(u_{1}\right)\right]\right| \\
\text { (Lemma 3.9) } & =\sup _{\left\|v_{s}-u_{s}\right\|_{w} \leq \bar{\alpha}^{s} C}\left|\mu_{0}\left[v_{s}-u_{s}\right]\right|
\end{aligned}
$$

Since $\left\|v_{s}-u_{s}\right\|_{w} \rightarrow 0$ as $s \rightarrow 0$, and by (ii), $\sup _{t>s}\left\|\mathcal{T}^{t-s}\left(v_{s}\right)-\mathcal{T}^{t-s}\left(u_{s}\right)\right\|_{w} \leq$ $A\left\|v_{s}-u_{s}\right\|_{w} \rightarrow 0$, the assertion holds. 
Let $\left\{c_{t}\right\}$ be a sequence of functions in $\mathscr{B}_{w}$ and $\mathcal{R}$ be a real-valued risk map. Define $\mathcal{F}_{t}(v):=c_{t}+\mathcal{R}(v), \mathcal{F}^{(0)}(v):=v$, and $\mathcal{F}^{(t+1)}(v):=\mathcal{F}_{t+1}\left(\mathcal{F}^{(t)}(v)\right), t=0,1,2, \ldots$

Lemma 3.13. Suppose Assumption 3.1 holds. Then for all $v, u \in \mathscr{B}_{w}$, (i) $\sup _{t \geq 1}\left\|\mathcal{F}^{(t)}(v)-\mathcal{F}^{(t)}(u)\right\|_{w}<\infty$ and (ii) $\lim _{t \rightarrow \infty} \frac{1}{t}\left\|\mathcal{F}^{(t)}(v)-\mathcal{F}^{(t)}(u)\right\|_{w}=0$.

Proof. Due to Assumption [3.1, by (3.3), we have $\overline{\mathcal{R} \sharp}(w) \leq \gamma w+K^{\prime}$, where $K^{\prime}:=\beta K+1-\gamma$. Thus $\left|\mathcal{F}^{(1)}(v)-\mathcal{F}^{(1)}(u)\right| \leq \overline{\mathcal{R}^{\sharp}}(|v-u|) \leq\|v-u\|_{w} \overline{\mathcal{R}^{\sharp}}(w) \leq$ $\|v-u\|_{w}\left(\gamma w+K^{\prime}\right)$. By induction w.r.t. $t$, we have for $t=2,3, \ldots$

$$
\begin{aligned}
& \left|\mathcal{F}^{(t)}(v)-\mathcal{F}^{(t)}(u)\right| \leq \overline{\mathcal{R}^{\sharp}}\left(\left|\mathcal{F}^{(t-1)}(v)-\mathcal{F}^{(t-1)}(u)\right|\right) \\
\leq & \|v-u\|_{w} \overline{\mathcal{R}^{\sharp}}\left(\gamma^{t-1} w+K^{\prime} \sum_{i=0}^{t-2} \gamma^{i}\right) \leq\|v-u\|_{w}\left(\gamma^{t} w+K^{\prime} \sum_{i=0}^{t-1} \gamma^{i}\right)
\end{aligned}
$$

which implies $\sup _{t>1}\left\|\mathcal{F}^{(t)}(v)-\mathcal{F}^{(t)}(u)\right\|_{w}<\infty$. (ii) is an immediate result of (i).

TheOREm 3.14. Suppose Assumption 3.1 holds. Then, for each $c \in \mathscr{B}_{w}$, (i) the Poisson equation (3.1) has one solution $(\rho, h) \in \mathbb{R} \times \mathscr{B}_{w}$, where $\rho$ is unique, and (ii) there exists a real-valued risk measure $\nu$ such that $\nu(c+\mathcal{R}(v))=\nu(v)+\rho, \forall v \in \mathscr{B}_{w}$.

Proof. (i) Define $\mathcal{T}_{c}(\cdot):=c+\mathcal{R}(\cdot)$. Then by Assumption 3.1 and Theorem 3.11 , the map $\mathcal{T}_{c}: \mathscr{B}_{w} \rightarrow \mathscr{B}_{w}$ is a contraction under $w$-seminorm, i.e., $\left\|\mathcal{T}_{c}(v)-\mathcal{T}_{c}(u)\right\|_{s, w} \leq$ $\bar{\alpha}\|v-u\|_{s, w}$, for some $\bar{\alpha} \in[0,1)$. In the following, we extend the fixed-point theorem w.r.t. span-seminorm (cf. p. 321 [1] for bounded $w$ ) to $w$-seminorm. Let $\widetilde{\mathscr{B}}_{w}=\mathscr{B}_{w} / \sim$ be the quotient space, which is induced by the equivalence relation $\sim$ on $\mathscr{B}_{w}$ defined by $v \sim u$ if and only if there exists some constant $C \in \mathbb{R}$ such that $v(x)-u(x)=C$ for all $x \in \mathbf{X}$, endowed with the quotient norm induced by the $w$-seminorm. For $v \in \mathscr{B}_{w}$, let $\tilde{v}$ be the corresponding equivalent class in $\tilde{\mathscr{B}}_{w}$ and $\widetilde{\mathcal{T}}_{c}: \widetilde{\mathscr{B}}_{w} \rightarrow \widetilde{\mathscr{B}}_{w}$ be the canonically induced map, i.e., $\widetilde{\mathcal{T}}_{c}(\tilde{v}):=\widetilde{\mathcal{T}_{c}(v)}, v \in \mathscr{B}_{w}$. Since $\mathcal{T}_{c}$ is a contraction w.r.t. $w$-seminorm on $\mathscr{B}_{w}, \widetilde{\mathcal{T}}_{c}$ is a contraction on $\widetilde{\mathscr{B}}_{w}$ and therefore has a unique fixed point. Conversely, it follows that the map $\mathcal{T}_{c}$ has a $w$-seminorm fixed point. In other words, there exists $h \in \mathscr{B}_{w}$ such that $\left\|\mathcal{T}_{c}(h)-h\right\|_{s, w}=0$ and $\rho_{c}:=\mathcal{T}_{c}(h)-h$ is a constant.

Next we show that such $\rho_{c}$ is unique. Define $\mathcal{T}_{c}^{t}(\cdot):=\mathcal{T}_{c}\left(\mathcal{T}_{c}^{t-1}(\cdot)\right), t=2,3, \ldots$ Suppose there exits another solution $\left(\rho^{\prime}, h^{\prime}\right) \in \mathbb{R} \times \mathscr{B}_{w}$ to the Poisson equation. Then $\mathcal{T}_{c}^{t}\left(h^{\prime}\right)=t \rho+h^{\prime}$ and $\mathcal{T}_{c}^{t}(h)=t \rho_{c}+h$. However, by Lemma 3.13 (ii), $\frac{1}{t} \| \mathcal{T}_{c}^{t}\left(h^{\prime}\right)-$ $\mathcal{T}_{c}^{t}(h) \|_{w} \rightarrow 0$, which implies that $\lim _{t \rightarrow \infty} \frac{1}{t}\left\|t \rho^{\prime}+h^{\prime}-t \rho_{c}-h\right\|_{w}=0$. Hence, $\rho^{\prime}=\rho_{c}$.

(ii) Let $h \in \mathscr{B}_{w}$ be a solution to the Poisson equation and $\mu_{0} \in \mathscr{M}_{w}$ is a probability measure. Define $\mathcal{D}_{c}(\cdot):=\mathcal{T}_{c}(\cdot)-\rho_{c}$ and $\mu_{t}(\cdot):=\mu_{0}\left(\mathcal{D}_{c}^{t}(\cdot)\right)$. Then we have $\forall v \in \mathscr{B}_{w}$,

$$
\mathcal{T}_{c}^{t}(v)-\mathcal{T}_{c}^{t}(h)-\left(\mathcal{T}_{c}^{s}(v)-\mathcal{T}_{c}^{s}(h)\right)=\mathcal{T}_{c}^{t}(v)-\mathcal{T}_{c}^{s}(v)-(t-s) \rho_{c}=\mathcal{D}_{c}^{t}(v)-\mathcal{D}_{c}^{s}(v) .
$$

Due to (3.9), the condition (ii) in Lemma 3.12 holds. Hence, setting $u=h$ in Lemma 3.12 we obtain that

$$
\lim _{s \rightarrow \infty} \sup _{t \geq s}\left|\mu_{0}\left[\mathcal{T}_{c}^{t}(v)-\mathcal{T}_{c}^{t}(h)\right]-\mu_{2}\left[\mathcal{T}_{c}^{s}(v)-\mathcal{T}_{c}^{s}(h)\right]\right|=0
$$

which implies for each $v \in \mathscr{B}_{w}$,

$$
\lim _{s \rightarrow \infty} \sup _{t \geq s} \mu_{0}\left[\mathcal{D}_{c}^{t}(v)-\mathcal{D}_{c}^{s}(v)\right]=\lim _{s \rightarrow \infty} \sup _{t \geq s}\left[\mu_{t}(v)-\mu_{s}(v)\right]=0 .
$$

Hence $\mu_{t}$ converges to a mapping $\mu_{\infty}: \mathscr{B}_{w} \rightarrow \mathbb{R}$ satisfying $\mu_{\infty}\left(\mathcal{D}_{c}(v)\right)=\mu_{\infty}(v)$, $\forall v \in \mathscr{B}_{w}$. Other other hand, it is easy to see that for each $t, \mu_{t}$ is a real-valued risk measure except the axiom of centralization. Hence, $\mu_{\infty}(c+\mathcal{R}(v))=\mu_{\infty}(v)+\rho_{c}$. Finally, by setting $\nu(v):=\mu_{\infty}(v)-\mu_{\infty}(0)$, we obtain the required risk measure. 


\section{Applying Risk Measures to MCPs.}

4.1. Risk Maps for MCPs. We define the risk maps with controls as follows

Definition 4.1. $\mathcal{R}(v \mid x, a)$ (simply written as $\mathcal{R}$ ) is said to be a risk map on an $\operatorname{MCP}(\mathbf{X}, \mathbf{A},\{\mathbf{A}(x) \mid x \in \mathbf{X}\}, Q)$, if (i) for each $(x, a) \in \mathbf{K}, \mathcal{R}(\cdot \mid x, a): \mathscr{B}_{w} \rightarrow \mathbb{R}$ is a real-valued risk measure; and (ii) for each $v \in \mathscr{B}_{w}, \mathcal{R}(v \mid \cdot)$ is a real-valued $\mathcal{B}(\mathbf{K})$ measurable function. Furthermore, we define for any $\pi \in \Delta$

$$
\mathcal{R}^{\pi}(v \mid x):=\int_{\mathbf{A}(x)} \pi(d a \mid x) \mathcal{R}(v \mid x, a) .
$$

For convenience, we sometimes write $\mathcal{R}_{x, a}(v):=\mathcal{R}(v \mid x, a)$ and $\mathcal{R}_{x}^{\pi}(v):=\mathcal{R}^{\pi}(v \mid x)$.

Remark. Note that since risk maps are subjective representations of objectives transition probabilities, as in the above definition, $\mathcal{R}$ depends always on the transition model $Q$ of the underlying MCP. It is obvious that the transition kernel $P^{\pi}$ defined in (2.1) is a valid risk map. Thus, the concept of a risk map is a generalization of the conditional expectation. (4.1) in fact assumes that $\mathcal{R}^{\pi}$ is linear to the policy $\pi$, which simplifies the optimization problem and is one of the conditions that guarantee the existence of one optimal deterministic policy ("optimal selector").

In the mathematical finance literature, there exist various ways to extend the CRM to a temporal structure (see e.g. 20, 9, 38] and references therein). The definition is usually selected based on applications. To compare their subtle differences are out of the scope of this paper. The risk maps defined here are similar to the risk measure generators in [9] and are implicitly Markovian and time-homogeneous (see also [38), since $\mathcal{R}$ defined above depends merely on the most recent state and action but not the whole history. The risk maps used in this paper are assumed to be Markovian, since in the MCP-framework the underlying stochastic process is Markov, while the assumption of time-homogeneity is due to the fact that since we consider the infinite-horizon criteria (see (5.2) and (5.3) ), as in the literature of MCPs, stationary optimal policies are expected. Hence, to comply with the MCP-framework, it is sufficient to construct an operator which replaces the conditional expectation determined by the transition model $Q$ and policy $\pi$.

4.2. Risk Preference. It is important to know how to judge the risk-preference of one specific risk map according to the properties of the map. In the following, we introduce the correspondence between the risk-preference and the convexity (concavity) by an intuitive example. Recall that in economics risk represents uncertainties 77. Thus there is no risk if there is no uncertainty. Suppose we are given two options, the first one is $(v, p ; u, 1-p), 0<p<1$, to obtain outcome (reward or cost) $v$ with probability $p$ and outcome $u$ with probability $1-p$; the second one is to obtain outcome $p \cdot v+(1-p) \cdot u$ with probability 1 . The first one is riskier than the second one in the sense that though both options have the same mean outcome, the first one includes uncertainty $p$ (and $1-p$ ) whereas the outcome is certain in the second option. Thus, if the first one is preferred, the agent is risk-seeking; if the second one is preferred, the agent is risk-averse.

In our framework, the preference is determined by the value $\mathcal{R}_{x, a}(v)$. Note that we want to minimize risk. If the $\mathcal{R}$ is concave w.r.t. $v$ at some $(x, a) \in \mathbf{K}$,

$$
p \mathcal{R}_{x, a}(v)+(1-p) \mathcal{R}_{x, a}(u) \leq \mathcal{R}_{x, a}(p v+(1-p) u), \forall p \in[0,1], v, u \in \mathscr{B}
$$

the riskier option is preferred. Thus, $\mathcal{R}$ is intuitively risk-seeking at $(x, a)$. Conversely, if $\mathcal{R}$ is convex w.r.t. $v$ at $(x, a)$, the agent is risk-averse at $(x, a)$. 
Remark. The categorization depends on the objective. In the expected utility theory [24, the objective is to maximize utilities. Therefore, the categorization is opposite: concavity means risk-averse and convexity suggests risk-seeking. Several existing risk measures (see Section 4.3) in the literature confirm also the above defined categorizations from intuitive sense.

In some cases, it is reasonable to take different risk-preferences at different situations, e.g. at "safe" states the bolder policies are taken to obtain high uncertain reward, while at "dangerous" states the conservative policies are applied to avoid high uncertain cost. Even when risk-averse policies are applied everywhere, the degree of risk-averse at different states can be also different. Within our framework, mixed risk-preferences can be easily modeled due to the following lemma.

Lemma 4.2. Let $\mathcal{R}, \mathcal{R}^{\prime}$ be two risk maps. For any $B \in \mathcal{B}(\mathbf{K})$, we define

$$
\tilde{\mathcal{R}}(v \mid x, a):=\mathbf{1}_{B}(x, a) \mathcal{R}(v \mid x, a)+\mathbf{1}_{B^{C}}(x, a) \mathcal{R}^{\prime}(v \mid x, a),
$$

where $\mathbf{1}_{B}(\cdot)$ denotes the indicator function and $B^{C}$ is the complementary set of $B$. Then $\tilde{\mathcal{R}}$ is also a risk map.

Proof. Obviously for each $(x, a) \in \mathbf{K}, \tilde{\mathcal{R}}(\cdot \mid x, a)$ is a risk measure, since both $\mathcal{R}(\cdot \mid x, a)$ and $\mathcal{R}^{\prime}(\cdot \mid x, a)$ are risk measures. Given $v \in \mathscr{B}_{w}$, since $\mathcal{R}(v \mid \cdot)$ and $\mathcal{R}^{\prime}(v \mid \cdot)$ are both real-valued $\mathcal{B}(\mathbf{K})$-measurable functions, it follows that $\mathcal{R}(v \mid \cdot)$ is also a real-valued $\mathcal{B}(\mathbf{K})$-measurable function.

For instance, suppose $\mathcal{R}$ and $\mathcal{R}^{\prime}$ are maps of everywhere risk-averse and riskseeking respectively. Then, by above lemma, $\tilde{\mathcal{R}}$ would be the risk map that is riskaverse at $B$ but risk-seeking at $B^{C}$ (e.g. $B:=\{k \in \mathbf{K} \mid c(k) \geq 0\}$ ). Therefore, the lemma gives us the power to model mixed risk-preferences by constructing new maps from existing maps whose properties have been well investigated.

4.3. Examples. There exist several important risk maps in the literature of economics, mathematical finance and control theory. Most of them can be adapted to the framework we introduced above. Since most of the literature consider merely bounded space $\mathscr{B}$ (or essentially bounded space) except the classical MCPs, in this section, we restrict ourselves to $\mathscr{B}$ when introducing definitions of different maps. Note that we focus mostly on the literature of optimal control and operation research. For more examples in mathematical finance, we refer to Chapter 4 of [22].

Classical MCPs. $\mathcal{R}_{x, a}(v):=\mathbb{E}_{x, a}^{Q}[v]=\int_{\mathbf{X}} Q(\mathrm{~d} y \mid x, a) v(y)$. It is easy to verify that it is coherent and linear to $v$ (therefore risk-neutral).

Entropic map. The name is taken from the literature of CRM 21. It is intensely also researched in the field of optimal control [11, 3, 4, 16, 12, 19, 26, 33, 5].

$$
\mathcal{R}_{x, a}(v):=\frac{1}{\lambda} \log \mathbb{E}_{x, a}^{Q}[\exp (\lambda v)]=\frac{1}{\lambda} \log \left\{\int_{\mathbf{X}} Q(\mathrm{~d} y \mid x, a) \exp (\lambda v(y))\right\}
$$

where the risk-sensitive parameter $\lambda \in \mathbb{R}$ controls the risk-preference of $\mathcal{R}$ : if $\lambda>0, \mathcal{R}$ is everywhere convex and therefore everywhere risk-averse; if $\lambda<0, \mathcal{R}$ is everywhere concave and therefore everywhere risk-seeking. It can be also shown that

$$
\lim _{\lambda \rightarrow 0} \frac{1}{\lambda} \log \left\{\int_{y} Q(\mathrm{~d} y \mid x, a) \exp (\lambda v(y))\right\}=\int_{y} Q(\mathrm{~d} y \mid x, a) v(y)
$$

which is equivalent to the classical MCPs. Besides, it has connection to the meanvariance tradeoff scheme via the Taylor expansion at $\lambda=0, \frac{1}{\lambda} \log \mathbb{E} \exp (\lambda Z)=\mathbb{E} Z+$ $\lambda \operatorname{Var}(Z)+O\left(\lambda^{2}\right)$ where $Z$ denotes an arbitrary bounded random variable. Suppose 
that risk is measured by variance. The objective is to minimize risk $\mathcal{R}^{\pi}$. Therefore, if $\lambda>0$, the variance is avoided, the agent is risk-averse. On the contrary, if $\lambda<0$, the variance is preferred, the agent is intuitively risk-seeking. These intuitions coincide the categorization based on the convexity (concavity) of $\mathcal{R}$.

Robust risk maps. Iyengar (2005) 31 introduced the framework of robust dynamic programming, by which he argues that in some applications the transition model $Q$ cannot be inferred exactly. Instead, he employs a set of transition probabilities, $\mathcal{P}$, which contains all possible "ambiguous" transition kernels. In order to gain the "robustness", the worst cost is considered, adapted in our framework,

$$
\mathcal{R}_{x, a}(v):=\sup _{Q(\cdot \mid x, a) \in \mathcal{P}_{x, a}} \mathbb{E}_{x, a}^{Q} v=\sup _{Q(\cdot \mid x, a) \in \mathcal{P}_{x, a}} \int_{y \in \mathbf{X}} Q(\mathrm{~d} y \mid x, a) v(y) .
$$

It is apparent that $\mathcal{R}$ is coherent. We can verify that $\mathcal{R}$ is everywhere convex and therefore risk-averse, which coincides the intuition that the worst scenario is considered. One special case of the robust dynamic programming was the minimax control (see e.g. [12]), which also considers the worst scenario with finite state space, $\mathcal{R}_{x, a}(v):=\max _{Q(y \mid x, a)>0} v(y)$. It is also notable that each coherent risk map has one dual presentation of the form (4.3) under some regularity conditions for the set $\mathcal{P}_{x, a}$ (see e.g. 13] for essentially bounded spaces and [43] for unbounded ones).

Mean-semideviation trade-off. [35, 40] This risk map considers only the trade-off between the one-step conditional mean and semideviation rather than the deviation of the whole Markov chain [42, 18].

$$
\mathcal{R}_{x, a}(v):=\mathbb{E}_{x, a}^{Q}[v]+\lambda\left[\mathbb{E}_{x, a}^{Q}\left(v-\mathbb{E}_{x, a}^{Q}[v]\right)_{+}^{r}\right]^{1 / r}
$$

where $r \geq 1$ and $\lambda \in \mathbb{R}$ denotes the risk-preference parameter which controls the risk preference of $R$ : if $\lambda>0, R$ is risk-averse; if $\lambda<0, R$ is risk-seeking. Setting $r=2$, this map can be viewed as an approximation of the mean-variance tradeoff scheme defined in 18 .

Prospect theory and Choquet integral. The idea is based on non-additive measures (Denneberg, 1994, 14]) and capacities (Choquet, 1953, 10]) that generalize standard probability measures (p.m.). Non-additive p.m. (for definition see [14]) can be analogously extended to fit the MCP framework with conditional non-additive p.m. $\Phi$, which satisfies that (i) for each $(x, a) \in \mathbf{K}, \Phi(\cdot \mid x, a)$ is a non-additive p.m. and (ii) for each $B \in \mathcal{B}(\mathbf{X}), \Phi(B \mid \cdot)$ is $\mathcal{B}(\mathbf{K})$-measurable. Then we define the conditional Choquet integral as

$$
\int_{\mathbf{X}}^{C h} v(y) \Phi(\mathrm{d} y \mid x, a):=\int_{-\infty}^{0}[\Phi(v(y)>t \mid x, a)-1] \mathrm{d} t+\int_{0}^{\infty} \Phi(v(y)>t \mid x, a) \mathrm{d} t .
$$

It is easy to see that for each conditional non-additive p.m. $\Phi$, Choquet integral is a homogeneous risk map but not necessarily convex everywhere. The well-known prospect theory 45 in behavioral economics, which is applied to interpret human behaviors of mixed risk-preferences, can be in fact represented as a Choquet integral 32, 45. In a recent study, Chateauneuf and Cohen (2008) [6] apply the Choquet integral to model the subjective expected utility [41].

5. Optimal Risk-sensitive Control. Suppose $\mathcal{R}$ is a risk map and $\boldsymbol{\pi} \in \Pi_{M}$. Analogous to the classical MCPs, we consider the following three objectives: (a) $T$-stage total risk,

$$
J_{T}(x, \boldsymbol{\pi}):=c^{\pi_{0}}(x)+\mathcal{R}_{x}^{\pi_{0}}\left(c^{\pi_{1}}+\mathcal{R}^{\pi_{1}}\left(c^{\pi_{2}}+\ldots+\mathcal{R}^{\pi_{T-1}}\left(c^{\pi_{T}}\right) \ldots\right)\right)
$$


(b) the discounted total risk as

$$
J_{\alpha}(x, \boldsymbol{\pi}):=\lim _{T \rightarrow \infty} J_{\alpha, T}(x, \boldsymbol{\pi})
$$

where the discounted $T$-stage risk is defined as follows,

$$
J_{\alpha, T}(x, \boldsymbol{\pi}):=c^{\pi_{0}}(x)+\alpha \mathcal{R}_{x}^{\pi_{0}}\left(c^{\pi_{1}}+\alpha \mathcal{R}^{\pi_{1}}\left(c^{\pi_{2}}+\ldots+\alpha \mathcal{R}^{\pi_{T-1}}\left(c^{\pi_{T}}\right) \ldots\right)\right)
$$

and (c) the average risk

$$
J(x, \boldsymbol{\pi}):=\limsup _{T \rightarrow \infty} \frac{1}{T} J_{T}(x, \boldsymbol{\pi}), \boldsymbol{\pi} \in \Pi_{M}, x \in \mathbf{X} .
$$

The optimal control problems for above three risk-sensitive objectives are to minimize the risk among all Markov policies

$$
J_{T}^{*}(x):=\inf _{\boldsymbol{\pi} \in \Pi_{M}} J_{T}(x, \boldsymbol{\pi}), \quad J_{\alpha}^{*}(x):=\inf _{\boldsymbol{\pi} \in \Pi_{M}} J_{\alpha}(x, \boldsymbol{\pi}) \text {, and } \quad J^{*}(x):=\inf _{\boldsymbol{\pi} \in \Pi_{M}} J(x, \boldsymbol{\pi}) .
$$

In the rest of this section, for convenience, all the three objectives can be considered as functions on $\mathbf{X}$ within the space $\mathscr{B}_{w}$ by using the notations $J_{T}(\boldsymbol{\pi}), J_{\alpha}(\boldsymbol{\pi})$ and $J(\boldsymbol{\pi})$, as well as $J_{T}^{*}, J_{\alpha}^{*}$ and $J^{*}$.

The finite-stage total risk can be solved by dynamic programming (cf. [38]). In this paper, we focus on the discounted total risk and average risk, which will be discussed in detail in Section 5.1 and 5.2 respectively.

In the rest of this section, we shall frequently use the following operators

$$
\mathcal{F}_{\alpha}^{\pi}(v):=c^{\pi}+\alpha \mathcal{R}^{\pi}(v), \quad \mathcal{F}_{\alpha}(v):=\inf _{\boldsymbol{\pi} \in \Pi_{M}} \mathcal{F}_{\alpha}^{\pi}(v), \quad v \in \mathscr{B}_{w}
$$

where $\alpha \in[0,1]$. If $\alpha=1$, we simply write them as $\mathcal{F}^{\pi}$ and $\mathcal{F}$ accordingly. The operators $\left(\mathcal{F}_{\alpha}^{\pi}\right)^{t}, t \in \mathbb{N}$, is defined iteratively as $\left(\mathcal{F}_{\alpha}^{\pi}\right)^{0}(v):=v$, and $\left(\mathcal{F}_{\alpha}^{\pi}\right)^{t}(v):=$ $\mathcal{F}_{\alpha}^{\pi}\left(\left(\mathcal{F}_{\alpha}^{\pi}\right)^{t-1}(v)\right), t=1,2, \ldots$, while $\mathcal{F}_{\alpha}^{t}$ is defined analogously.

Analogous to classical MCPs, we need further assumptions to guarantee the existence of the "selector" in the optimization problem.

Assumption 5.1. For each $x \in \mathbf{X}$,

(a) the cost function $c(x, a)$ is lower semi-continuous on $\mathbf{A}(x)$,

(b) the action space $\mathbf{A}(x)$ is compact, and

(c) the function $u^{\prime}(x, a):=\mathcal{R}_{x, a}(u)$ is continuous in $a \in \mathbf{A}(x)$ for any $u \in \mathscr{B}_{w}$.

Proposition 5.1. Suppose $\mathcal{R}$ is a risk map satisfying Assumption 5.1. Then, for all $v \in \mathscr{B}_{w}$ and $x \in \mathbf{X}$, there exists a deterministic policy $f \in \Delta_{D}$, such that for any $\alpha \in[0,1], c^{f}(x)+\alpha \mathcal{R}^{f}(v \mid x)=\mathcal{F}_{\alpha}(v \mid x)=\inf _{\pi \in \Delta}\left\{c^{\pi}(x)+\alpha \mathcal{R}^{\pi}(v \mid x)\right\}$.

Proof. Apparently, $\mathcal{F}_{\alpha}(v \mid x)=\inf _{a \in \mathbf{A}(x)}\{c(x, a)+\alpha \mathcal{R}(v \mid x, a)\}$ for each $x \in \mathbf{X}$. By Assumption 5.1(a) and (c), the function $u(x, a):=c(x, a)+\alpha \mathcal{R}(v \mid x, a), \alpha \in[0,1]$, is lower semi-continuous in $a \in \mathbf{A}(x)$ for each $x \in \mathbf{X}$. Hence, by Assumption 5.1(b) and Lemma 8.3.8(a) [29], the optimal selector exists.

5.1. Discounted Total Risk. In economics, the time-discount is added to reflect the "time-value" of outcomes: the outcome to be gained in the future is less valuable than the same amount of outcome obtained now. It has similar effects when cost is concerned. Due to its good mathematical properties, exponential discounting scheme, where the cost $c_{t}$ is multiplied with the time-discount $\alpha^{t}$, is widely applied in 
economics, finance as well as in MCPs (see $S_{\alpha}$ in (2.2)). In fact, the natural extension of classical discounted MCPs is as follows,

$$
D_{\alpha}(\boldsymbol{\pi})=c^{\pi_{0}}+\mathcal{R}^{\pi_{0}}\left(\alpha c^{\pi_{1}}+\mathcal{R}^{\pi_{1}}\left(\alpha^{2} c^{\pi_{2}}+\ldots+\mathcal{R}^{\pi_{T-1}}\left(\alpha^{T} c^{\pi_{T}}+\ldots\right) \ldots\right)\right) .
$$

However, since the risk map $\mathcal{R}$ is not necessarily homogeneous, there might not exist a stationary policy when optimizing $D_{\alpha}$. Indeed, Chung \& Sobel (1987) 11] proved that for the entropic risk maps, which are not homogeneous, the optimal policy might not be stationary if $D_{\alpha}(\boldsymbol{\pi})$ is optimized w.r.t. $\boldsymbol{\pi}$, though $D_{\alpha}$ is well-defined if $\alpha \in[0,1)$.

In our definition, discount factor $\alpha$ is multiplied with $\mathcal{R}$, which has the same "time-discount" effect, where the risk rather than the immediate cost is discounted. Moreover, it is easy to see that, if $\mathcal{R}$ is homogeneous, $D_{\alpha}$ is equivalent to $J_{\alpha}$, the discounted total risk under our definition. Therefore, $D_{\alpha}$ defined for any homogeneous risk map is merely a special case of our definition. Specifically, the classical discounted MCP is indeed a special case of our defined discounted total risk, since it is homogeneous. Ruszczyński (2010) [38] used $D_{\alpha}$ as the objective function, which was solved by a value iteration algorithm, since merely the coherent risk maps (both homogeneous and convex) were considered. Besides, in the proof of the value iteration algorithm, he used the representation theorem, which is valid merely for coherent risk maps. On the contrary, we will see later that the objective $J_{\alpha}$ allows a value iteration algorithm for general risk maps. Therefore, we apply $J_{\alpha}$ rather than $D_{\alpha}$.

Let $\boldsymbol{\pi}=\left[\pi_{0}, \pi_{1}, \ldots\right] \in \Pi_{M}$ be one Markov policy. Using the operator $\mathcal{F}^{\pi}$ defined in (5.4), we have

$$
J_{\alpha, T}(\boldsymbol{\pi})=\mathcal{F}_{\alpha}^{\pi_{0}}\left(\mathcal{F}_{\alpha}^{\pi_{1}} \ldots \mathcal{F}_{\alpha}^{\pi_{T-1}}\left(c^{\pi_{T}}\right) \ldots\right)=\mathcal{F}_{\alpha}^{\pi_{0}}\left(\mathcal{F}_{\alpha}^{\pi_{1}} \ldots \mathcal{F}_{\alpha}^{\pi_{T-1}}\left(\mathcal{F}_{\alpha}^{\pi_{T}}(0)\right) \ldots\right) .
$$

We first show that under some assumption, the limit in (5.2) exists.

Assumption 5.2. There exist nonnegative constants $\bar{c}$ and $\bar{w}$, with $1 \leq \bar{w}<1 / \alpha$ and a weight function $w \geq 1$ such that $\forall x \in \mathbf{X}:(a) \sup _{a \in \mathbf{A}(x)}\|c(x, a)\| \leq \bar{c} w(x)$ and (b) $\sup _{a \in \mathbf{A}(x)} \overline{\mathcal{R}^{\sharp}}(w \mid x, a) \leq \bar{w} w(x)$, where $\overline{\mathcal{R}^{\sharp}}$ is defined in Section 3.1.

Applying Proposition 3.7 we obtain the following proposition.

Proposition 5.2. Suppose Assumption 5.2(b) holds. Then for all $\pi \in \Delta, v, u \in$ $\mathscr{B}_{w},\left\|\mathcal{F}_{\alpha}^{\pi}(v)-\mathcal{F}_{\alpha}^{\pi}(u)\right\|_{w} \leq \bar{w} \alpha\|v-u\|_{w}$.

Lemma 5.3. Suppose Assumption 5.2(a) and (b) holds. Then (i) $\lim _{T \rightarrow \infty} J_{\alpha, T}(\boldsymbol{\pi})$ exists in $\mathscr{B}_{w}$, for all $\boldsymbol{\pi} \in \Pi_{M}$, and (ii) $J_{\alpha, T}(\boldsymbol{\pi})=\lim _{T \rightarrow \infty} \mathcal{F}_{\alpha}^{\pi_{0}}\left(\mathcal{F}_{\alpha}^{\pi_{1}} \ldots \mathcal{F}_{\alpha}^{\pi_{T}}(v) \ldots\right)$, $\forall v \in \mathscr{B}_{w}$.

Proof. By Assumption $5.2(\mathrm{a}), c^{\pi} \in \mathscr{B}_{w}$ for all $\pi \in \Delta$. Iterating Proposition 5.2 .

$$
\begin{aligned}
& \left\|J_{\alpha, T+1}(\boldsymbol{\pi})-J_{\alpha, T}(\boldsymbol{\pi})\right\|_{w} \\
= & \left\|\mathcal{F}_{\alpha}^{\pi_{0}}\left(\mathcal{F}_{\alpha}^{\pi_{1}} \ldots \mathcal{F}_{\alpha}^{\pi_{T}}\left(c^{\pi_{T+1}}\right) \ldots\right)-\mathcal{F}_{\alpha}^{\pi_{0}}\left(\mathcal{F}_{\alpha}^{\pi_{1}} \ldots \mathcal{F}_{\alpha}^{\pi_{T-1}}\left(\mathcal{F}_{\alpha}^{\pi_{T}}(0)\right) \ldots\right)\right\|_{w} \\
\leq & (\bar{w} \alpha)^{T}\left\|c^{\pi_{T+1}}\right\|_{w} .
\end{aligned}
$$

By Assumption $5.2(\mathrm{a}), 0 \leq \bar{w} \alpha<1$. Thus $J_{\alpha, T+1}(\boldsymbol{\pi}) \rightarrow J_{\alpha, T}(\boldsymbol{\pi})$ and $\left\|J_{\alpha, T+1}(\boldsymbol{\pi})\right\|_{w}<$ $\frac{\bar{c}}{1-\bar{w} \alpha}$ is bounded and thus in $\mathscr{B}_{w}$. (ii) is straightforward by replacing $c^{\pi_{T+1}}$ with $v$ in (5.5).

Lemma 5.4. Suppose Assumption 5.2 and 5.1 hold. Then $\left\|\mathcal{F}_{\alpha}(v)-\mathcal{F}_{\alpha}(u)\right\|_{w} \leq$ $\bar{w} \alpha\|v-u\|_{w}, 0 \leq \bar{w} \alpha<1$.

Proof. By Proposition [5.1 the optimal selector $f^{*}$ always exists for all $v \in \mathscr{B}_{w}$. Let $f_{v}$ be the optimal selector for $v$ and $f_{u}$ be the optimal selector for $u$. Thus

$$
\mathcal{F}_{\alpha}(v)-\mathcal{F}_{\alpha}(u) \leq \mathcal{F}_{\alpha}^{f_{u}}(v)-\mathcal{F}_{\alpha}^{f_{u}}(u) \leq \bar{w} \alpha\|v-u\|_{w} w
$$


where the latter inequality is due to Proposition 5.2. On the other hand,

$$
\mathcal{F}_{\alpha}(u)-\mathcal{F}_{\alpha}(v) \leq \mathcal{F}_{\alpha}^{f_{v}}(u)-\mathcal{F}_{\alpha}^{f_{v}}(v) \leq \bar{w} \alpha\|v-u\|_{w} w .
$$

Hence, $\left\|\mathcal{F}_{\alpha}(v)-\mathcal{F}_{\alpha}(u)\right\|_{w} \leq \bar{w} \alpha\|v-u\|_{w}$.

Since $\mathcal{F}_{\alpha}$ is a contracting map and $\mathscr{B}_{w}$ is complete, by the Banach fixed point theorem, it has the unique fixed point in $\mathscr{B}_{w}$ such that

$$
v^{*}(x)=\mathcal{F}_{\alpha}\left(v^{*} \mid x\right)=\min _{a \in \mathbf{A}(x)}\left\{c(x, a)+\alpha \mathcal{R}\left(v^{*} \mid x, a\right)\right\} .
$$

The final task is to show that $v^{*}=J_{\alpha}^{*}$, the optimal discounted total risk.

Theorem 5.5. Suppose Assumption 5.2 and 5.1 hold. Then $v^{*}=J_{\alpha}^{*}$.

Proof. First, we show $v \geq \mathcal{F}_{\alpha} v$ implies $v \geq J_{\alpha}^{*}$. By Proposition [5.1] we assume that the optimal selector for $v$ is $f$. Hence, we obtain

$$
v \geq \mathcal{F}_{\alpha}^{f} v \geq \mathcal{F}_{\alpha}^{f} \mathcal{F}_{\alpha}^{f} v \geq \mathcal{F}_{\alpha}^{f} \mathcal{F}_{\alpha}^{f} \mathcal{F}_{\alpha}^{f}(v) \geq\left(\mathcal{F}_{\alpha}^{f}\right)^{\infty}(v)=J_{\alpha}\left(f^{\infty}\right) \geq J_{\alpha}^{*}
$$

where the equality is due to Lemma 5.3 (ii). Second, we show that $v \leq \mathcal{F}_{\alpha} v$ implies $v \leq J_{\alpha}^{*}$. Indeed, let $\boldsymbol{\pi}=\left[\pi_{0}, \pi_{1}, \ldots\right] \in \Pi_{M}$ be an arbitrary Markov random policy. Then, for all $\pi \in \Delta, v \leq \mathcal{F}_{\alpha} v \leq \mathcal{F}_{\alpha}^{\pi} v$. Hence,

$$
v \leq \mathcal{F}_{\alpha}^{\pi_{0}}(v) \leq \mathcal{F}_{\alpha}^{\pi_{0}} \mathcal{F}_{\alpha}^{\pi_{1}}(v) \leq \mathcal{F}_{\alpha}^{\pi_{0}} \mathcal{F}_{\alpha}^{\pi_{1}} \mathcal{F}_{\alpha}^{\pi_{2}}(v) \leq \mathcal{F}_{\alpha}^{\pi_{0}} \ldots \mathcal{F}_{\alpha}^{\pi_{T}}(v) \uparrow J_{\alpha}(\boldsymbol{\pi}) .
$$

The limit is due to Lemma [5.3(ii). Since $\pi$ can be arbitrarily chosen, it follows $v \leq \inf _{\boldsymbol{\pi} \in \Pi_{M}} J_{\alpha}(\boldsymbol{\pi})=J_{\alpha}^{*}$. Combining above two steps yields $v^{*}=J_{\alpha}^{*}$.

Corollary 5.6. Under Assumption 5.2 and 5.1, there exists a stationary deterministic policy $f^{*} \in \Delta_{D}$ such that $J_{\alpha}^{*}=J_{\alpha}\left(\left(f^{*}\right)^{\infty}\right)$.

5.2. Average Risk. In the first part of this section, we extend the Assumption 3.1 to the MCP-framework and compare it with assumptions in the existing literature of classical MCPs and entropic maps. In the second part, we prove the existence of solutions to the average risk optimality equation.

Assumption 5.3. There exists a function $W: \mathbf{X} \rightarrow[0, \infty)$ which is $\mathcal{B}(\mathbf{X})$ measurable and constants $K \geq 0, \gamma \in(0,1), \alpha \in(0,1)$ and a risk measure $\nu$ s.t. (i)

$$
\overline{\mathcal{R}}_{x, a}(W) \leq \gamma W(x)+K, \forall(x, a) \in \mathbf{K}
$$

where $\mathcal{R}_{x, a}^{\sharp}(W)$ is calculated on the space $\mathscr{B}_{w}=\mathscr{B}_{1+\beta W}, \beta:=\alpha_{0} / K$, for some $\alpha_{0} \in(0, \alpha)$, and (ii) for all $v \geq u \in \mathscr{B}_{w}$,

$$
\inf _{x \in B,(x, a) \in \mathbf{K}}\left\{\mathcal{R}_{x, a}(v)-\alpha \nu(v)-\mathcal{R}_{x, a}(u)+\alpha \nu(u)\right\} \geq 0
$$

where $B=\{x \in \mathbf{X}: W(x) \leq R\} \in \mathcal{B}(\mathbf{X})$ for some $R>2 K /(1-\gamma)$.

Comparison with classical MCPs. In this case, $\mathcal{R}_{x, a}(v)=\overline{\mathcal{R}}_{x, a}(v)=Q_{x, a}(v)$ and obviously (5.8) is equivalent to $\inf _{x \in B,(x, a) \in \mathbf{K}} Q_{x, a}(A) \geq \nu(A)$ for all $A \in \mathcal{B}(\mathbf{X})$, where $\nu$ becomes a probability measure. Therefore, Assumption 5.3 becomes the classical condition with a Lyapunov function, which has been widely used in the MCPs literature (see e.g. [30, 29, 46] and references therein) for studying the optimization problem of average costs with Borel spaces and unbounded costs, to ensure that for each deterministic policy $f \in \Delta_{D}$, the Markov chain with transition kernel $Q^{f}$ is $w$-weighted geometric ergodic (for definition see [34]). 
Comparison with entropic maps. The most widely studied risk map in MCPliterature is the entropic map (4.2). While most of them focus on finite or countable state spaces, only a few papers (see e.g. [16] and references therein) discussed the general state space model as we consider in this paper under the sup-norm $\|\cdot\|_{\infty}:=$ $\|\cdot\|_{w=1}$. Thus we restrict to $\mathscr{B}$. Furthermore, all of the MCP-literature applying the entropic maps consider merely risk-averse cases, i.e., $\lambda>0$. Hence, without loss of generality, we set $\lambda=1$. In the following, we connect the generalized Doeblin's condition stated in (5.8) with properties of kernel $Q$.

Proposition 5.7. Suppose there exists a set $C \in \mathcal{B}(\mathbf{K})$, a constant $\alpha>0$ and a probability $\nu$ such that $\inf _{k \in C} Q(\cdot \mid k) \geq \alpha \nu(\cdot)$. Then for all $v \geq u \in \mathscr{B}$ and $\|u\|_{\infty} \leq$ $M<\infty$, there exists a constant $\epsilon(M)>0$, such that, for all $v \geq u,\|u\|_{\infty} \leq M$,

$$
\inf _{k \in C}\left\{\mathcal{R}_{k}(v)-\mathcal{R}_{k}(u)-\epsilon(M) \nu(v)+\epsilon(M) \nu(u)\right\} \geq 0
$$

where $\mathcal{R}$ is the entropic risk map defined in (4.2).

Proof. Note that adding any constant valued function to $v$ will not change the inequality in (5.9). Hence, we assume that $-2 M \leq u \leq 0$. By Lemma 6.1 of [44, we have $g(k, u):=\frac{\exp (u)}{\int_{\mathbf{X}} \exp (u) d Q_{k}} \geq \exp (-2 M)$ is one subgradient in $\delta \mathcal{R}_{k}(u)$. Hence, by definition of subgradient and assumption

$$
\mathcal{R}_{k}(v)-\mathcal{R}_{k}(u) \geq \int(v-u) g d Q_{k} \geq \alpha \exp (-2 M) \int(v-u) d \nu .
$$

Setting $\epsilon(M)=\alpha \exp (-2 M)>0$, we obtain (5.9).

Note that though (5.9) is weaker than the generalized Doeblin's condition stated in (5.8), for the purpose of proving the existence of solutions to Poisson equation (5.10), it is sufficient (cf. Proposition 2.2 of [15]) to consider the functions $u$ upper bounded by some constant $M$ under sup-norm, if the cost function $c$ is upper bounded by $M$, i.e., $|c(k)| \leq M, \forall k \in \mathbf{K}$. In [15, the condition $\sup _{k, k^{\prime} \in \mathbf{K}, B \in \mathcal{B}(\mathbf{X})}\left|Q_{k}(B)-Q_{k^{\prime}}(B)\right| \leq \alpha$ is assumed. It is known that this condition implies (see e.g. [17]) the Doeblin's condition holds on the whole space $\mathbf{K}$, i.e., there exists a probability measure $\mu$ and a constant $\alpha^{\prime}>0$ such that $\inf _{k \in \mathbf{K}} Q(B \mid k) \geq \alpha^{\prime} \mu(B), \forall B \in \mathcal{B}(\mathbf{X})$. Hence, setting $W=1$ in (5.7), Assumption 5.3 holds. It means that the condition stated in 15] is covered by our assumption. However, it is still an open question whether our assumption is stronger than the assumption considered in the most recent paper [16.

From now on, we state proofs related to the average risk optimality equation.

Lemma 5.8. Suppose Assumption 5.1 and 5.3 hold. Then there exists $\bar{\alpha} \in[0,1)$ such that $\|\mathcal{F}(v)-\mathcal{F}(u)\|_{s, w} \leq \bar{\alpha}\|v-u\|_{s, w}$, for all $v, u \in \mathscr{B}_{w}$, where $\mathcal{F}$ is defined in (5.4).

Proof. Under Assumption [5.1, by Proposition [5.1, there exists a deterministic policy $f_{v}, f_{u} \in \Delta_{D}$ such that $\mathcal{F}(v)=\mathcal{F}^{f_{v}}(v)$ and $\mathcal{F}(u)=\mathcal{F}^{f_{u}}(u)$. Thus

$$
\begin{aligned}
\mathcal{F}(v)-\mathcal{F}(u) & \leq \mathcal{F}^{f_{u}}(v)-\mathcal{F}^{f_{u}}(u)=\mathcal{R}^{f_{u}}(v)-\mathcal{R}^{f_{u}}(u) \\
\text { and } \quad \mathcal{F}(u)-\mathcal{F}(v) & \leq \mathcal{F}^{f_{v}}(u)-\mathcal{F}^{f_{v}}(v)=\mathcal{R}^{f_{v}}(u)-\mathcal{R}^{f_{v}}(v)
\end{aligned}
$$

yield $\mathcal{F}_{x}(v)-\mathcal{F}_{x}(u)+\mathcal{F}_{y}(u)-\mathcal{F}_{y}(v) \leq \mathcal{R}_{x}^{f_{u}}(v)-\mathcal{R}_{x}^{f_{u}}(u)+\mathcal{R}_{y}^{f_{v}}(u)-\mathcal{R}_{y}^{f_{v}}(v)$ for all $x, y \in \mathbf{X}$. By Assumption 5.3 and repeating the proof in Theorem 3.11, we have

$$
\begin{aligned}
& \mathcal{F}_{x}(v)-\mathcal{F}_{x}(u)+\mathcal{F}_{y}(u)-\mathcal{F}_{y}(v) \\
\leq & \mathcal{R}_{x}^{f_{u}}(v)-\mathcal{R}_{x}^{f_{u}}(u)+\mathcal{R}_{y}^{f_{v}}(u)-\mathcal{R}_{y}^{f_{v}}(v) \leq \bar{\alpha}\|v-u\|_{s, w} d_{w}(x, y) .
\end{aligned}
$$


Switching $v$ and $u$, the l.h.s of the inequality does not change. Thus we have $\mid \mathcal{F}_{x}(v)-$ $\mathcal{F}_{x}(u)+\mathcal{F}_{y}(u)-\mathcal{F}_{y}(v) \mid \leq \bar{\alpha}\|v-u\|_{s, w} d_{w}(x, y)$.

Above lemma shows that the operator $\mathcal{F}: \mathscr{B}_{w} \rightarrow \mathscr{B}_{w}$ is a contraction under the $w$-seminorm. Hence, by Theorem 3.14 (i) we immediate obtain the following theorem.

TheOREM 5.9. Suppose Assumption 5.1, 5.2(a) and5.3 hold. Then there exists a unique $\rho^{*} \in \mathbb{R}$ and $h \in \mathscr{B}_{w}$ satisfying the average risk optimality equation (AROE)

$$
\rho^{*}+h(x)=\mathcal{F}(h \mid x)=\inf _{a \in \mathbf{A}(x)}\{c(x, a)+\mathcal{R}(h \mid x, a)\} .
$$

Theorem 5.10. Suppose Assumption 5.1, 5.2(a) and 5.3 hold. Then $\rho=$ $J^{*}(x)=J\left(x, f^{\infty}\right)$ for all $x \in \mathbf{X}$, where $\rho$ is the solution to the AROE ([5.100) and $f$ denotes the optimal selector in the r.h.s of the AROE.

Proof. Under Assumption 5.1, 5.2(a) and 5.3. the existence of one solution to the AROE is guaranteed by Theorem [5.9] By Proposition [5.1, the optimal selector $f$ exists. Thus we assume $\mathcal{F}(h)=\mathcal{F}^{f}(h)$. Due to the AROE,

$$
\left(\mathcal{F}^{f}\right)^{t}(h)=\left(\mathcal{F}^{f}\right)^{t-1}(\rho+h)=t \rho+h \quad \Rightarrow \quad \lim _{t \rightarrow \infty} \frac{1}{t}\left\|\left(\mathcal{F}^{f}\right)^{t}(h)-\rho\right\|_{w}=0 .
$$

On the other hand, for any $v \in \mathscr{B}_{w}$, by Lemma 3.13 (ii), $\frac{1}{t}\left\|\left(\mathcal{F}^{f}\right)^{t}(v)-\left(\mathcal{F}^{f}\right)^{t}(h)\right\|_{w} \rightarrow 0$ implies that $J\left(x, f^{\infty}\right)=\lim _{t \rightarrow \infty} \frac{1}{t}\left(\mathcal{F}^{f}\right)_{x}^{t}(0)=\rho, \forall x \in \mathbf{X}$.

Next we prove that $\rho \leq J(x, \boldsymbol{\pi})$ for all $\boldsymbol{\pi} \in \Pi_{M}$ and $x \in \mathbf{X}$. In fact, let $\boldsymbol{\pi}=$ $\left[\pi_{0}, \pi_{1}, \ldots\right]$ be an arbitrary Markov policy. Then by Lemma 3.13 (ii), for all $v \in \mathscr{B}_{w}$,

$$
\lim _{t \rightarrow \infty} \frac{1}{t} \|\left(\mathcal{F}^{\pi_{0}}\left(\mathcal{F}^{\pi_{1}} \ldots \mathcal{F}^{\pi_{t}}(v)\right)-\mathcal{F}^{\pi_{0}}\left(\mathcal{F}^{\pi_{1}} \ldots \mathcal{F}^{\pi_{t}}(0)\right) \|_{w}=0\right.
$$

By definition $h \leq \mathcal{F}^{\pi}(h)-\rho, \forall \pi \in \Delta$. Iterating this inequality yields

$$
h \leq \mathcal{F}^{\pi_{0}}\left(\mathcal{F}^{\pi_{1}}\left(\ldots \mathcal{F}^{\pi_{t-1}}(h)\right)\right)-t \rho \Rightarrow \limsup _{t \rightarrow \infty} \frac{1}{t} \mathcal{F}^{\pi_{0}}\left(\mathcal{F}^{\pi_{1}}\left(\ldots \mathcal{F}^{\pi_{t-1}}(h)\right)\right) \geq \rho .
$$

Note that by definition $J(\boldsymbol{\pi})=\lim \sup _{t \rightarrow \infty} \frac{1}{t} \mathcal{F}^{\pi_{0}}\left(\mathcal{F}^{\pi_{1}}\left(\ldots \mathcal{F}^{\pi_{t-1}}(0)\right)\right)$ and by setting $v=h$ in (5.11), we obtain $J(\boldsymbol{\pi})=\limsup _{t \rightarrow \infty} \frac{1}{t} \mathcal{F}^{\pi_{0}}\left(\mathcal{F}^{\pi_{1}}\left(\ldots \mathcal{F}^{\pi_{t-1}}(h)\right)\right)$. Hence, (5.12) implies $\rho \leq J(\boldsymbol{\pi})$. It follows that $\rho \leq \inf _{\boldsymbol{\pi} \in \Pi_{M}} J(\boldsymbol{\pi})=J^{*}$. Since $f^{\infty}$ is a valid Markov policy in $\Pi_{M}, \rho=J^{*}=J\left(f^{\infty}\right)$.

6. One Example with Mean-semideviation. We present one example by applying the mean-semideviation map defined in (4.4) with $r=2$ and $\lambda \in(0,1)$ which has not be considered as an average risk objective in MCP-literature yet. Consider a 1-dimensional simple linear model

$$
X_{t+1}=b\left(A_{t}\right) X_{t}+N_{t}
$$

where $N_{t}$ is i.i.d white noise and $b$ is a real-valued measurable function on $\mathcal{B}(\mathbf{A})$. Hence the transition kernel in this MCP is simply $Q(d y \mid x, a)=\frac{1}{\sqrt{2 \pi}} e^{-\frac{(y-b(a) x)^{2}}{2}} d y$. Assume that $\sup _{a \in \mathbf{A}}|b(a)|=\epsilon<1$ and set $k=(x, a)$ for simplicity. Recall that $\mathbb{E}_{k}^{Q} v:=\int_{\mathbf{X}=\mathbb{R}} Q(d y \mid k) v(y)$.

First, we show that $W(x)=x^{2}$ is a weight function satisfying (5.7). Indeed, since the mean-semideviation map $\mathcal{R}$ is coherent if $\lambda \in(0,1), \overline{\mathcal{R}^{\sharp}}=\mathcal{R}$.

$$
\begin{aligned}
\mathcal{R}_{k}(W) & =\mathbb{E}_{k}^{Q} W+\lambda \sqrt{\mathbb{E}_{k}^{Q}\left(W-\mathbb{E}_{k}^{Q} W\right)_{+}^{2}} \leq \mathbb{E}_{k}^{Q} W+\lambda \sqrt{\mathbb{E}_{k}^{Q}\left(W-\mathbb{E}_{k}^{Q} W\right)^{2}} \\
& =b^{2}(a) x^{2}+1+\lambda \sqrt{2+4 b^{2}(a) x^{2}} \leq \epsilon^{2} x^{2}+1+\lambda \sqrt{2+4 \epsilon^{2} x^{2}}
\end{aligned}
$$


Note that, for any $\gamma \in\left(\epsilon^{2}, 1\right)$, the function $t \mapsto\left(\epsilon^{2}-\gamma\right) t+1+\lambda \sqrt{2+4 \epsilon^{2} t}$ is concave in $[0, \infty)$ and its maximum is attained at some constant $K$. Thus,

$$
\epsilon^{2} x^{2}+1+\lambda \sqrt{2+4 \epsilon^{2} x^{2}} \leq \gamma x^{2}+K \quad \Rightarrow \quad \mathcal{R}_{k}(W) \leq \gamma W(x)+K
$$

holds for all $k \in \mathbf{K}$. Thus, the weight function is $w(x)=1+\beta W(x)=1+\beta x^{2}$ with some $\beta>0$.

Next, we check the condition stated in (5.8). Note that if $v \in \mathscr{B}_{w}$, then $\mathbb{E}_{k}^{Q}\left[v^{2}\right] \leq$ $\|v\|_{w}^{2} \mathbb{E}_{k}^{Q}\left[1+\beta y^{2}\right]^{2}<\infty$ for each $k$. Hence, $L^{2}(Q(\cdot \mid k)) \supset \mathscr{B}_{w}$. It is known that (see e.g. [44)

$$
g(k, u)= \begin{cases}1 & \text { if } u \text { is constant } \\ 1-\lambda \frac{\mathbb{E}_{k}^{Q}\left[\left(u-\mathbb{E}_{k}^{Q} u\right)_{+}\right]-\left(u-\mathbb{E}_{k}^{Q} u\right)_{+}}{\sqrt{\mathbb{E}_{k}^{Q}\left[\left(u-\mathbb{E}_{k}^{Q} u\right)_{+}^{2}\right]}} & \text { otherwise }\end{cases}
$$

is one subgradient defined on $L^{2}(Q(\cdot \mid k))$ for $u \in L^{2}(Q(\cdot \mid k)) \supset \mathscr{B}_{w}$. Note that since

$$
\mathbb{E}_{k}^{Q}\left[\left(u-\mathbb{E}_{k}^{Q} u\right)_{+}\right]-\left(u-\mathbb{E}_{k}^{Q} u\right)_{+} \leq \mathbb{E}_{k}^{Q}\left[\left(u-\mathbb{E}_{k}^{Q} u\right)_{+}\right] \leq \sqrt{\mathbb{E}_{k}^{Q}\left[\left(u-\mathbb{E}_{k}^{Q} u\right)_{+}^{2}\right]},
$$

$g(k, u) \geq 1-\lambda>0$, for $\lambda \in(0,1)$. Besides, it is easy to check that for each $k$ and $u \in \mathscr{B}_{w}$,

$$
\mathbb{E}_{k}^{Q}[|g(k, u)| w]=\mathbb{E}_{k}^{Q}[g(k, u) w] \leq \mathbb{E}_{k}^{Q}\left[\left(1+\lambda \frac{\left(u-\mathbb{E}_{k}^{Q} u\right)_{+}}{\sqrt{\mathbb{E}_{k}^{Q}\left[\left(u-\mathbb{E}_{k}^{Q} u\right)_{+}^{2}\right]}}\right) w\right]<\infty
$$

Hence $g(k, u) \in \delta \mathcal{R}_{k}(u), \forall k \in \mathbf{K}, u \in \mathscr{B}_{w}$. On the other hand, the MCP defined in (6.1) satisfies that for any closed set $C_{m}:\{k \in \mathbf{K}|| x \mid \leq m\}, m>0$, the classical Doeblin's condition holds (cf. p. 380 of [34]). Hence, by Proposition 3.10, the generalized Doeblin's condition holds. Together with (6.2), Assumption 5.3 holds.

Acknowledgments. The authors would like to thank the reviewers and the associated editor for careful readings and constructive suggestions, which lead to an improvement of the presentation of the paper.

\section{REFERENCES}

[1] A. Arapostathis, V.S. Borkar, E. Fernández-Gaucherand, M.K. Ghosh, and S.I. Marcus. Discrete-time controlled markov processes with average cost criterion: a survey. SIAM Journal on Control and Optimization, 31(2):282-344, 1993.

[2] P. Artzner, F. Delbaen, J.M. Eber, and D. Heath. Coherent measures of risk. Mathematical Finance, 9(3):203-228, 1999.

[3] G. Avila-Godoy and E. Fernández-Gaucherand. Controlled Markov chains with exponential risk-sensitive criteria: modularity, structured policies and applications. In Decision and Control, 1998. Proceedings of the 37th IEEE Conference on, volume 1, pages 778-783. IEEE, 1998.

[4] V.S. Borkar and S.P. Meyn. Risk-sensitive optimal control for Markov decision processes with monotone cost. Mathematics of Operations Research, pages 192-209, 2002.

[5] R. Cavazos-Cadena. Optimality equations and inequalities in a class of risk-sensitive average cost Markov decision chains. Mathematical Methods of Operations Research, 71(1):47-84, 2010 . 
[6] A. Chateauneuf and M. Cohen. Cardinal extensions of the EU model based on the Choquet integral. Decision-making Process, pages 401-433, 2008.

[7] J.P. Chavas. Risk Analysis in Theory and Practice. Academic Press, 2004.

[8] P. Cheridito, F. Delbaen, and M. Kupper. Dynamic monetary risk measures for bounded discrete-time processes. Electronic Journal of Probability, 11(3):57-106, 2006.

[9] P. Cheridito and M. Kupper. Composition of time-consistent dynamic monetary risk measures in discrete time. International Journal of Theoretical and Applied Finance, 14(1):137-162, 2011.

[10] G. Choquet. Theory of capacities. In Annales de l'institut Fourier, volume 5, 1953.

[11] K.J. Chung and M.J. Sobel. Discounted MDPs: distribution functions and exponential utility maximization. SIAM Journal on Control and Optimization, 25:49, 1987.

[12] S.P. Coraluppi and S.I. Marcus. Mixed risk-neutral/minimax control of discrete-time, finitestate Markov decision processes. Automatic Control, IEEE Transactions on, 45(3):528-532, 2000 .

[13] F. Delbaen. Coherent risk measures on general probability spaces. Advances in Finance and Stochastics Essays in Honour of Dieter Sondermann, pages 1-37, 2000.

[14] D. Denneberg. Non-additive Measure and Integral. Kluwer Academic Publishers, 1994.

[15] G.B. Di Masi and L. Stettner. Risk-sensitive control of discrete-time Markov processes with infinite horizon. SIAM Journal on Control and Optimization, 38:61, 1999

[16] G.B. Di Masi and L. Stettner. Infinite horizon risk sensitive control of discrete time Markov processes under minorization property. SIAM Journal on Control and Optimization, 46(1):231, 2008.

[17] R.L. Dobrushin. Central limit theorem for non-stationary Markov chains I. Theory Prob. Appl., pages 65-80, 1956.

[18] J.A. Filar, LCM Kallenberg, and H.M. Lee. Variance-penalized Markov decision processes. Mathematics of Operations Research, pages 147-161, 1989.

[19] W.H. Fleming and D. Hernández-Hernández. Risk sensitive control of finite state machines on an infinite horizon. I. In Decision and Control, 1997., Proceedings of the 36th IEEE Conference on, volume 4, pages 3407-3412. IEEE, 1997.

[20] H. Föllmer and I. Penner. Convex risk measures and the dynamics of their penalty functions. Statistics \& Decisions, 24(1/2006):61-96, 2006.

[21] H. Föllmer and A. Schied. Convex measures of risk and trading constraints. Finance and Stochastics, 6(4):429-447, 2002.

[22] H. Föllmer and A. Schied. Stochastic finance, volume 27 of de Gruyter Studies in Mathematics. Walter de Gruyter \& Co., Berlin, extended edition, 2004.

[23] S. Gaubert and J. Gunawardena. The Perron-Frobenius theorem for homogeneous, monotone functions. Transactions American Mathematical Society, 356(12):4931-4950, 2004.

[24] C. Gollier. The Economics of Risk and Time. The MIT Press, 2004.

[25] M. Hairer and J.C. Mattingly. Yet another look at harris' ergodic theorem for Markov chains. In Seminar on Stochastic Analysis, Random Fields and Applications VI, pages 109-117. Springer, 2011.

[26] D. Hernández-Hernández and S.I. Marcus. Risk sensitive control of Markov processes in countable state space. Systems \& control letters, 29(3):147-155, 1996.

[27] O. Hernández-Lerma. Adaptive Markov Control Processes, volume 79. Springer, 1989.

[28] O. Hernández-Lerma and J.B. Lasserre. Discrete-time Markov Control Processes: Basic Optimality Criteria. Springer, 1996.

[29] O. Hernández-Lerma and J.B. Lasserre. Further Topics on Discrete-Time Markov Control Processes. Springer Verlag, 1999.

[30] O. Hernández-Lerma, R. Montes-De-Oca, and R. Cavazos-Cadena. Recurrence conditions for Markov decision processes with Borel state space: a survey. Annals of Operations Research, 28(1):29-46, 1991.

[31] G.N. Iyengar. Robust dynamic programming. Mathematics of Operations Research, pages 257-280, 2005.

[32] D. Kahneman and A. Tversky. Prospect theory: an analysis of decision under risk. Econometrica: Journal of the Econometric Society, pages 263-291, 1979.

[33] S.I. Marcus, E. Fernández-Gaucherand, D. Hernández-Hernandez, S. Coraluppi, and P. Fard. Risk sensitive Markov decision processes. Progress in Systems and Control Theory, 22:263$280,1997$. 
[34] S.P. Meyn and R.L. Tweedie. Markov chains and stochastic stability. Communications and Control Engineering Series. Springer-Verlag London Ltd., London, 1993.

[35] W. Ogryczak and A. Ruszczyński. From stochastic dominance to mean-risk models: Semideviations as risk measures. European Journal of Operational Research, 116(1):33-50, 1999.

[36] M.L. Puterman. Markov Decision Processes: Discrete Stochastic Dynamic Programming. John Wiley \& Sons, Inc., 1994.

[37] B. Roorda, J.M. Schumacher, and J. Engwerda. Coherent acceptability measures in multiperiod models. Mathematical Finance, 15(4):589-612, 2005.

[38] A. Ruszczyński. Risk-averse dynamic programming for Markov decision processes. Mathematical Programming, pages 1-27, 2010.

[39] A. Ruszczyński and A. Shapiro. Conditional risk mappings. Mathematics of Operations Research, 31(3):544-561, 2006.

[40] A. Ruszczyński and A. Shapiro. Optimization of risk measures. Probabilistic and Randomized Methods for Design under Uncertainty, pages 119-157, 2006.

[41] L.J. Savage. The Foundations of Statistics. Dover Publications, 1972.

[42] M.J. Sobel. The variance of discounted Markov decision processes. Journal of Applied Probability, pages 794-802, 1982.

[43] G. Svindland. Convex risk measures beyond bounded risks. PhD thesis, Ludwig-MaximiliansUniversität München, 2009.

[44] G. Svindland. Subgradients of law-invariant convex risk measures on $L^{1}$. Statistics $\&$ Secisions, 27(2):169-199, 2009

[45] A. Tversky and D. Kahneman. Advances in prospect theory: cumulative representation of uncertainty. Journal of Risk and Uncertainty, 5(4):297-323, 1992.

[46] O. Vega-Amaya. The average cost optimality equation: a fixed point approach. Bol. Soc. Mat. Mexicana, 9(1):185-195, 2003. 